\title{
Light emission by accelerated electric, toroidal and anapole dipolar sources
}

\author{
V. Savinov \\ Optoelectronics Research Centre and Centre for Photonic Metamaterials, \\ University of Southampton, Southampton SO17 1BJ, UK
}

\begin{abstract}
Emission of electromagnetic radiation by accelerated particles with electric, toroidal and anapole dipole moments is analyzed. It is shown that ellipticity of the emitted light can be used to differentiate between electric and toroidal dipole sources, and that anapoles, elementary neutral non-radiating configurations, which consist of electric and toroidal dipoles, can emit light under uniform acceleration. The existence of non-radiating configurations in electrodynamics implies that it is impossible to fully determine the internal makeup of the emitter given only the distribution of the emitted light. Here we demonstrate that there is a loop-hole in this 'inverse source problem'. Our results imply that there may be a whole range of new phenomena to be discovered by studying the electromagnetic response of matter under acceleration.
\end{abstract}

\section{INTRODUCTION}

Electromagnetic radiation is produced by oscillating and accelerating charges and currents. The converse, however, is not true. There exists a wide class of configurations composed of oscillating charges and currents which emit no electromagnetic fields. These are known as the nonradiating configurations [1]. The nonradiating configurations are not merely a mathematical curiosity. Their existence directly implies that it is impossible to deduce the internal composition of a charge-current configuration given only information about the fields it emits. In practice, this means that if, given a field distribution, a suitable source charge-current configuration can be found, it will not be unique, since one can always add a nonradiating configuration to it without changing the emitted field. This feature of Maxwell's equations, also known as the inverse source problem [2-4], impacts many branches of science where light is used to interrogate distant or otherwise inaccessible objects, from medical imaging, to astronomy and radar science [4]. Here we will show that neutral nonradiating configurations can, in fact, be made to radiate by using acceleration. This is a loop-hole in the inverse source problem. Unlike other techniques for characterizing localized oscillating chargecurrent configurations, such as near-field microscopy for example [5], our solution is universal since it only relies on properties of Minkowski spacetime and Maxwell's Equations in vacuum.

The key to understanding the general properties of nonradiating configurations is to consider their elementary building blocks - anapoles. Dynamic anapoles are point-like nonradiating configurations that consist of copositioned and co-aligned electric and toroidal dipoles [68], as shown in Fig. 1a. The electric dipole is the usual infinitesimal electromagnetic excitation created by two separated charges [9]. The toroidal dipole is another fundamental point-like excitation that can be represented as current flowing on the surface of a torus (see Fig. 1a). Introduced by Ya. B. Zeldovich in 1957 [6] in the context of nuclear and particle physics, toroidal dipoles have since (a)

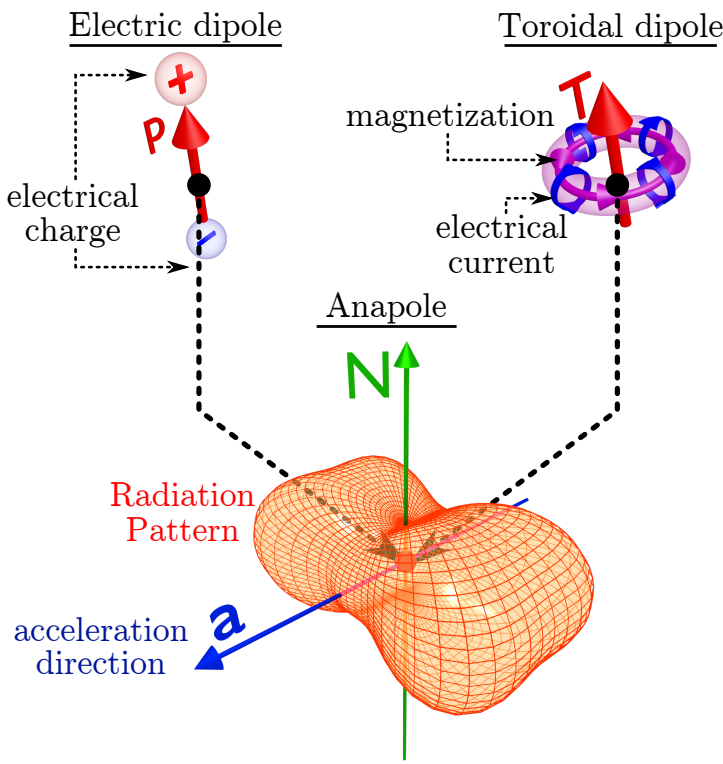

Figure 1. Radiation pattern of a point-like nonradiating configuration (anapole) subjected to acceleration. (a) Electric and toroidal dipoles that together make up the anapole. The electric dipole $(\boldsymbol{p})$ corresponds to two separated opposite charges. The toroidal dipole $(\boldsymbol{T})$ corresponds to a loop of magnetization, or, equivalently, to current flowing along the meridians of a torus. (b) Radiation pattern (radius represents power per solid angle) of a dynamic anapole with moment $\boldsymbol{N}$, accelerating with constant acceleration $\boldsymbol{a}$.

been discovered in a number of systems ranging from atomic nuclei to solid state physics and artificial composite media (for a recent review see [8]).

An important feature of monochromatic electromagnetic radiation in vacuum is that double-curls of both the electric and magnetic fields are proportional to the fields themselves (i.e. $\nabla \times \nabla \times \boldsymbol{E}=k^{2} \boldsymbol{E}$ for electric field, where $k$ is the wavenumber). This symmetry leads to the emission of electric and toroidal dipoles being identical anywhere outside the source region [8]. Indeed, this is why these two dipoles can be combined to create a 
point-like nonradiating configuration (anapole). For a long time, dynamic, i.e. time-varying, anapoles remained a theoretical concept. Their existence was first demonstrated in a microwave metamaterial [10], and has since been repeated in a variety of man-made systems in various domains of the electromagnetic spectrum [8, 11-15]. From the general properties of nonradiating configurations it is relatively simple to show that any nonradiating configuration can be regarded as a collection of anapoles $[1,16,17]$. Therefore, anapoles can be used as a convenient model to understand the general properties of all nonradiating configurations. The focus of this work is on anapole as well as electric and toroidal dipoles in non-inertial motion.

It is well-known that motion, and especially noninertial motion, changes the fields produced by charged particles [9]. The same applies to the fields due to neutral particles with various multipole moments. The motion of particles with conventional electric and magnetic multipole moments was first investigated more than 50 years ago [18-21]. However the properties of particles with toroidal dipole and anapole moments in non-inertial motion have so far been overlooked (see App. C). Here, for the first time, we present a rigorous treatment of radiation produced by a uniformly accelerated neutral particle with an anapole moment (anapole particle). In the process, we also develop the first treatment of the radiation produced by an accelerated particle with a toroidal dipole moment. We show that despite being nonradiating when at rest (or in inertial motion, see App. D), anapole particles do produce radiation when subjected to acceleration. By extension, this implies that all accelerated 'nonradiating configurations' do, in fact, radiate. Finally, we show that acceleration provides a way of unambiguously differentiating between electric and toroidal dipole excitations based solely on far-field radiation, thus overcoming the limitation set by the inverse-source problem.

The structure of the paper is as follows. The conventional notion of point-particles with electric, toroidal and anapole dipole moments is generalized to a covariant description in terms of four-currents, in Sec. II. The general approach of obtaining far-field radiation from accelerating point-particles is discussed in Sec. III. The results of these two sections are combined to obtain the farfield radiation patterns of accelerated dipoles, in Sec. IV. The results are analyzed and summarized in Sec. V. Appendix contains additional material on the derivations as well as a section on the notation used in the paper (see App. A).

\section{COVARIANT DESCRIPTION OF ELECTRIC, TOROIDAL AND ANAPOLE DIPOLE POINT-PARTICLES}

This section provides the definitions of the electric, toroidal and anapole dipole electromagnetic excitations in terms of charge and current density, and then generalizes these definitions to four-currents of point-particles in arbitrary motion.

As is shown in Fig. 1, an anapole corresponds to a superposition of electric and toroidal dipoles. The charge $(\rho)$ and current $(\boldsymbol{J})$ densities of an anapole particle stationary in the lab-frame are [8]:

$$
\begin{gathered}
\rho(t, \boldsymbol{r})=-\boldsymbol{\nabla} \cdot\left(\boldsymbol{p} \delta^{(3)}\right) \\
\boldsymbol{J}(t, \boldsymbol{r})=\dot{\boldsymbol{p}} \delta^{(3)}+\boldsymbol{\nabla} \times \boldsymbol{\nabla} \times\left(c \boldsymbol{T} \delta^{(3)}\right) \\
\boldsymbol{T}=\boldsymbol{N}, \quad \boldsymbol{p}=\dot{\boldsymbol{N}} / c
\end{gathered}
$$

Here, $c$ is the speed of light, $t$ and $\boldsymbol{r}$ are time and the position of the observer, whilst $\boldsymbol{p}=\boldsymbol{p}(t)$ and $\boldsymbol{T}=\boldsymbol{T}(t)$ are the electric and toroidal dipole moments (respectively). The three-dimensional delta function is denoted with $\delta^{(3)}=\delta^{(3)}(\boldsymbol{r}-\overline{\boldsymbol{r}})$, where $\overline{\boldsymbol{r}}$ is the position of the point-particle. The time-derivative is denoted by 'overdot', i.e. $d \boldsymbol{p} / d t \equiv \dot{\boldsymbol{p}}$. Apart from defining anapole excitation, Eqs. $(1,2)$ also provide the definition for the charge and current density of a point-like electric dipole (obtained by setting $\boldsymbol{T} \rightarrow 0$ ) and a point-like toroidal dipole (obtained by setting $\boldsymbol{p} \rightarrow 0$ ). We now return to the definition of the anapole. To make an anapole, the electric and toroidal dipoles in the equations above have to be linked. A convenient way to do so is to introduce an 'anapole dipole moment' $\boldsymbol{N}$, defined by Eq. (3).

Before proceeding, we introduce a convenient terminology. Clearly, the anapole defined in Eqs. (1-3) is just one possible kind of a nonradiating configuration. A suitable super-position of electric and toroidal quadrupoles, for example, can also lead to nonradiating configurations, which will differ from the anapole introduced above. We therefore will refer to the excitations defined in Eqs. (1-3) as 'anapole dipole' throughout this paper.

The definition provided in Eqs. (1-3) is ill-suited for calculations involving moving and accelerating sources, one therefore needs to generalize it to four-current of an electric/toroidal/anapole dipole. This is accomplished in the next three sub-sections. Sub-sections II A and II B provide the correct expressions for the four-current of a point-particle with electric dipole moment and toroidal dipole moment (respectively). Superposition of these two four-currents, which corresponds to an anapole, is given the Sub-section II C. 


\section{A. Four-current of a point-particle with electric dipole moment}

Here we consider the four-current $\left(J^{(p) \mu}\right)$ due to a point-particle with electric dipole moment $(\boldsymbol{p})$. That is, we consider the four-current due to a point-particle in arbitrary motion, which, when characterized in its instantaneous rest frame, has only the electric dipole moment $\boldsymbol{p}$. The four-current can be derived in a number of ways, including simply considering a pair of co-moving opposite charges that oscillate about a point $\bar{x}^{\mu}$, which is the four-position of the electric dipole particle. Fortunately, point-particles with electric dipole moment have already been considered in the literature [20]. The four-current due to electric dipole point-particle is:

$$
J^{(p) \mu}=\int d \tau c^{2} p^{\alpha} \hat{\tau}^{\beta} \delta_{\alpha \beta}^{\mu \eta} \nabla_{\eta} \delta^{(4)}(x-\bar{x})
$$

Where $\tau$ is the proper time of the particle, $\bar{x}^{\mu}=\bar{x}^{\mu}(\tau)$ is the four-position of the particle, $\hat{\tau}^{\alpha}=\left(d \bar{x}^{\mu} / d \tau\right) / c$ is the unit-length four-vector tangent to the particle worldline, $\nabla_{\eta}$ is the covariant derivative, $\delta_{\alpha \beta}^{\mu \eta}=\delta_{\alpha}^{\mu} \delta_{\beta}^{\eta}-\delta_{\beta}^{\mu} \delta_{\alpha}^{\eta}$ is the generalized Kroenecker delta [22], and $p^{\alpha}$ is the four-vector related to the electric dipole moment of the particle. One can demonstrate that Eq. (4) is the correct expression by considering its equivalent in the instantaneous rest frame of the particle. Let frame $\tilde{S}$ be an inertial reference frame in which the electric dipole particle is at rest at proper time $\tau=\tau_{0}$ which corresponds to time $\tilde{t}=0$ in $\tilde{S}$. Assume that velocity of the particle in $\tilde{S}$ remains negligible over the time-period $\tilde{t}=-\frac{\epsilon}{2} \ldots \frac{\epsilon}{2}$. Within this period the four-current due to electric dipole point-particle in $\tilde{S}$ is:

$$
\begin{aligned}
\tilde{J}^{(p) \mu} & =\int_{-\epsilon / 2}^{\epsilon / 2} \frac{d \tilde{t}^{\prime}}{\gamma} c^{2} \tilde{p}^{\alpha} \tilde{\hat{\tau}}^{\beta} \tilde{\delta}_{\alpha \beta}^{\mu \eta} \tilde{\nabla}_{\eta} \Lambda \delta^{(4)}(\tilde{x}-\tilde{\bar{x}}) \\
\tilde{J}^{(p) \mu} & =\int_{-\epsilon / 2}^{\epsilon / 2} d \tilde{t}^{\prime} c^{2} \tilde{p}^{\alpha} \tilde{\delta}_{\alpha 0}^{\mu \eta} \tilde{\nabla}_{\eta} \delta^{(4)}(\tilde{x}-\tilde{\bar{x}})
\end{aligned}
$$

Where $\tilde{\bar{x}}^{\mu}=(c \tilde{t}, \tilde{\overline{\boldsymbol{r}}})$ is the four-position of the particle, $\gamma=d \tilde{t} / d \tau=1 / \sqrt{1-\dot{\overline{\boldsymbol{r}}}^{2} / c^{2}}$ is the Lorentz factor, $\Lambda=\operatorname{det}(\partial(\tilde{x}) / \partial(x))$ is the Jacobian due to changing coordinates from lab-frame to $\tilde{S}$. Since $\tilde{S}$ is the instantaneous rest-frame $\gamma=1$ and $\dot{\hat{\tau}}^{\beta}=\tilde{\delta}_{0}^{\beta}$ (i.e. temporal axis of $\tilde{S}$ is tangent to particle world line at $\tilde{t}=0$ ). We also assume that the the lab-frame and $\tilde{S}$ only differ by a boost, thus $\Lambda=1$.

Due to anti-symmetry of $\tilde{\delta}_{\alpha 0}^{\mu \eta}$ the four-vector $\tilde{p}^{\alpha}$ can be defined as space-like without any loss of generality: $\tilde{p}^{\alpha}=(0, \boldsymbol{p})^{\alpha}$. The charge density then becomes:

$$
\begin{aligned}
\tilde{\rho} & =\tilde{J}^{(p) 0} / c=\int_{-\epsilon / 2}^{\epsilon / 2} d \tilde{t}^{\prime} c \tilde{p}^{\alpha} \tilde{\delta}_{\alpha 0}^{0 \eta} \tilde{\nabla}_{\eta} \delta\left(c\left(\tilde{t}-\tilde{t}^{\prime}\right)\right) \delta^{(3)}(\tilde{\boldsymbol{r}}-\tilde{\tilde{\boldsymbol{r}}}) \\
& =-\int_{-\epsilon / 2}^{\epsilon / 2} d \tilde{t}^{\prime} c \tilde{p}^{\alpha} \tilde{\nabla}_{\alpha} \delta\left(c\left(\tilde{t}-\tilde{t}^{\prime}\right)\right) \delta^{(3)}(\tilde{\boldsymbol{r}}-\tilde{\boldsymbol{r}}) \\
& =-\boldsymbol{p} \cdot \tilde{\nabla} \delta^{(3)}\left(\tilde{\boldsymbol{r}}-\tilde{\boldsymbol{r}}_{0}\right)=-\boldsymbol{p} . \tilde{\nabla} \delta^{(3)}
\end{aligned}
$$

Which corresponds to charge density of a point-particle with electric dipole moment $\boldsymbol{p}$ (Eq. (1)). Above, $\tilde{\overline{\boldsymbol{r}}}_{0}$ denotes the position of the particle at time $\tilde{t}=0$ in frame $\tilde{S}$. The current density is $(i=1,2,3)$ :

$$
\begin{aligned}
\tilde{\boldsymbol{J}}^{i} & =\int_{-\epsilon / 2}^{\epsilon / 2} d \tilde{t}^{\prime} c^{2} \tilde{p}^{\alpha} \tilde{\delta}_{\alpha 0}^{i \eta} \tilde{\nabla}_{\eta} \delta\left(c\left(\tilde{t}-\tilde{t}^{\prime}\right)\right) \delta^{(3)}(\tilde{\boldsymbol{r}}-\tilde{\boldsymbol{r}}) \\
& =\int_{-\epsilon / 2}^{\epsilon / 2} d \tilde{t}^{\prime} c^{2} \tilde{p}^{i} \tilde{\nabla}_{0} \delta\left(c\left(\tilde{t}-\tilde{t}^{\prime}\right)\right) \delta^{(3)}(\tilde{\boldsymbol{r}}-\tilde{\overline{\boldsymbol{r}}}) \\
& =\int_{-\epsilon / 2}^{\epsilon / 2} d \tilde{t}^{\prime} c \tilde{p}^{i}\left(\tilde{t}^{\prime}\right) \partial_{\tilde{t}} \delta\left(c\left(\tilde{t}-\tilde{t}^{\prime}\right)\right) \delta^{(3)}(\tilde{\boldsymbol{r}}-\tilde{\overline{\boldsymbol{r}}}) \\
\tilde{\boldsymbol{J}} & =\left[\partial_{\tilde{t}}(\boldsymbol{p} \delta(\tilde{\boldsymbol{r}}-\tilde{\tilde{\boldsymbol{r}}}))\right]_{\tilde{t}=0}=\dot{\boldsymbol{p}} \delta^{(3)}-\boldsymbol{p}\left(\dot{\tilde{\overline{\boldsymbol{r}}}} \cdot \tilde{\tilde{\nabla}} \delta^{(3)}\right)=\dot{\boldsymbol{p}} \delta^{(3)}
\end{aligned}
$$

Note that by definition, the velocity of the pointparticle is negligible in $\tilde{S}$ during $\tilde{t}=-\epsilon / 2 \ldots \epsilon / 2$ so $\dot{\tilde{\boldsymbol{r}}}(0)=\dot{\overline{\boldsymbol{r}}}_{0}=0$. The above expression agrees with the current density due to point-particle with electric dipole $\boldsymbol{p}$ (Eq. (2) with $\boldsymbol{T}=0$ ). It follows that Eq. (4) is the four-current of a point-particle which can be described as electric dipole point-particle in its instantaneous rest frame $(\tilde{S})$ at any point in its history. The four-vector $p^{\mu}$ is given by $p^{\mu}=\frac{\partial x^{\mu}}{\partial \tilde{x}^{\nu}}(0, \boldsymbol{p})^{\nu}$, where $\tilde{S}$ is the instantaneous rest-frame of the particle and $\boldsymbol{p}$ is the electric dipole moment of the particle in $\tilde{S}$.

\section{B. Four-current of a point-particle with toroidal dipole moment}

For toroidal dipole, we adopt an approach similar to Sec. II A, the expression for the four-current of a pointparticle with toroidal dipole moment is first stated:

$$
J^{(T) \mu}=\int d \tau c^{2} T^{\gamma} \hat{\tau}^{\sigma} \hat{\tau}_{\eta} \delta_{\gamma \sigma \rho}^{\mu \eta \alpha} g^{\rho \beta} \nabla_{\alpha} \nabla_{\beta} \delta^{(4)}(x-\bar{x})
$$

and then it is shown that this expression does reduce to Eq. (2) (with $\boldsymbol{p}=\mathbf{0}$ ) in the instantaneous rest-frame of the particle. Above $\hat{\tau}_{\eta}=g_{\eta \nu} \hat{\tau}^{\nu}$, where $g_{\eta \nu}=\operatorname{diag}(1,-1,-1,-1)$ is the metric tensor and $g^{\rho \beta}$ is the inverse metric tensor. The four-vector $T^{\gamma}$ is related to the toroidal dipole moment of the point-particle.

As in Sec. II A, one goes into instantaneous rest frame 
$\tilde{S}$ of the particle, and uses $d \tau=d \tilde{t}, \tilde{\hat{\tau}}^{\sigma}=\tilde{\delta}_{0}^{\sigma}, \tilde{\hat{\tau}}_{\eta}=\tilde{\delta}_{\eta}^{0}$ :

$$
\begin{aligned}
\tilde{J}^{(T) \mu} & =\int_{-\epsilon / 2}^{\epsilon / 2} d \tilde{t}^{\prime} c^{2} \tilde{T}^{\gamma} \tilde{\delta}_{\gamma 0 \rho}^{\mu 0 \alpha} \tilde{g}^{\rho \beta} \tilde{\nabla}_{\alpha} \tilde{\nabla}_{\beta} \delta^{(4)}(\tilde{x}-\tilde{\bar{x}}) \\
\tilde{\rho} & =\tilde{J}^{(T) 0} / c=0 \\
\tilde{\boldsymbol{J}}^{(T) i} & =c(0, \boldsymbol{T})^{\gamma} \tilde{\delta}_{\gamma 0 \rho}^{i 0 \alpha} \tilde{g}^{\rho \beta} \tilde{\nabla}_{\alpha} \tilde{\nabla}_{\beta} \delta^{(3)}\left(\tilde{\boldsymbol{r}}-\tilde{\overline{\boldsymbol{r}}}_{0}\right) \\
& =c\left(\begin{array}{c}
-T_{x} \tilde{\nabla}^{2}+(\boldsymbol{T} . \tilde{\nabla}) \tilde{\partial}_{x} \\
-T_{y} \tilde{\nabla}^{2}+(\boldsymbol{T} . \tilde{\nabla}) \\
-T_{z} \tilde{\nabla}^{2}+(\boldsymbol{T} . \tilde{\nabla}) \tilde{\partial}_{z}
\end{array}\right) \delta^{(3)}\left(\tilde{\boldsymbol{r}}-\tilde{\overline{\boldsymbol{r}}}_{0}\right) \\
& =\tilde{\boldsymbol{\nabla}} \times \tilde{\nabla} \times c \boldsymbol{T} \delta^{(3)}
\end{aligned}
$$

Above, the anti-symmetry of $\delta_{\gamma \sigma \rho}^{\mu \eta \alpha}=\delta_{\gamma}^{\mu} \delta_{\sigma \rho}^{\eta \alpha}-\delta_{\sigma}^{\mu} \delta_{\gamma \rho}^{\eta \alpha}-$ $\delta_{\rho}^{\mu} \delta_{\sigma \gamma}^{\eta \alpha}$ ensures that $\tilde{J}^{(T) 0}=0$ and that $\tilde{T}^{0}$ has no effect on the four-current, hence one can set $\tilde{T}^{0}=0$. Thus the four-current in Eq. (5) corresponds to pointparticle which has toroidal dipole moment $\boldsymbol{T}$ in its instantaneous rest frame at all times (compare with Eq. (2) when $\boldsymbol{p}=0)$. As with electric dipole, the four-vector $T^{\gamma}=\frac{\partial x^{\mu}}{\partial \tilde{x}^{\nu}}(0, \boldsymbol{T})^{\nu}$ where $\tilde{S}$ is the instantaneous rest frame and $\boldsymbol{T}$ is the toroidal dipole moment of the point-particle in $\tilde{S}$.

\section{Four-current of a point-particle with anapole moment}

Combining the previous results in Eq. $(4,5)$ the fourcurrent of the anapole point-particle is:

$$
\begin{aligned}
J^{\mu}\left(x^{\nu}\right)=\int d \tau( & \left(c \frac{d N^{\gamma}}{d \tau} \hat{\tau}^{\sigma} \delta_{\gamma \sigma}^{\mu \alpha} \nabla_{\alpha} \delta^{(4)}+\right. \\
& \left.+c^{2} N^{\gamma} \hat{\tau}^{\sigma} \hat{\tau}_{\eta} \delta_{\gamma \sigma \rho}^{\mu \eta \alpha} g^{\rho \beta} \nabla_{\alpha} \nabla_{\beta} \delta^{(4)}\right)
\end{aligned}
$$

The four-vector $N^{\gamma}$ is such that in the instantaneous rest-frame of the particle $(\tilde{S})$ it has value $\tilde{N}^{\gamma}=(0, \boldsymbol{N})^{\gamma}$, where $\boldsymbol{N}$ is the anapole moment of Eq. (3).

To the best of our knowledge, this is the first time the covariant description of either the toroidal dipole or anapole has been presented.

\section{RADIATION FROM MOVING POINT-PARTICLE MULTIPOLES}

In this section we will show how the radiation field due to arbitrary point-dipoles can be evaluated in a straightforward fashion. As shown by several authors [18-20], four-currents due to moving point-dipoles can be expressed as linear combinations of derivatives of deltafunctions integrated along the world-line of the pointparticle:

$$
J^{\mu}(x)=\int d \tau K^{\mu \alpha_{1} \ldots \alpha_{n}} \nabla_{\alpha_{1}} \ldots \nabla_{\alpha_{n}} \delta^{(4)}(x-\bar{x}(\tau))
$$

Where $K^{\mu \alpha_{1} \ldots \alpha_{n}}$ is a tensor that depends on proper time $\tau$ and $\bar{x}^{\mu}(\tau)$ is the world-line of the point-particle. Indeed, the four-currents for electric (Eq. (4)) and toroidal (Eq. (5)) point-dipoles can be expressed in the form of Eq. (7). The four-potential $\left(A^{\mu}=(\phi / c, \boldsymbol{A})^{\mu}\right)$ due to four-current in Eq. (7) is given by $\partial_{\mu} \partial^{\mu} A^{\eta}=\mu_{0} J^{\eta}$, where $\mu_{0}$ is vacuum permeability [9]. Substituting the above current density one can find the retarded solution for the four-potential [9], and from that the electromagnetic tensor:

$$
\begin{aligned}
F_{\mu \eta}= & \delta_{\mu \eta}^{\gamma \xi} \nabla_{\gamma} A_{\xi} \\
= & \frac{\mu_{0}}{2 \pi} \delta_{\mu \eta}^{\gamma \xi} g_{\xi \kappa} \nabla_{\gamma} \nabla_{\alpha_{1}} \ldots \nabla_{\alpha_{n}}[ \\
& \left.\int d \tau K^{\kappa \alpha_{1} \ldots \alpha_{n}} \Theta\left(X^{0}\right) \delta(X \cdot X)\right]
\end{aligned}
$$

Where $X^{\eta}=x^{\eta}-\bar{x}^{\eta}$ is a four-vector connecting the four-position of the observer at $x^{\eta}=(c t, \boldsymbol{r})^{\eta}$ and the four-position of the point-particle at $\bar{x}^{\eta}=(c \bar{t}, \overline{\boldsymbol{r}})^{\eta}$. The Heaviside function $\Theta\left(X^{0}\right)=\Theta(c(t-\bar{t}))$ enforces causality. We will bear this constraint in mind and omit $\Theta\left(X^{0}\right)$ in what is to follow (i.e. we will restrict our consideration to $t>\bar{t}$, which implies an upper limit on the proper time integral $\int d \tau \rightarrow \int^{\tau(t)} d \tau$, which we will also omit for clarity).

In principle Eq. (8) can be evaluated in full generality, but the resulting expression tend to become very cumbersome very fast. Instead we offer a short-cut which is capable of pulling out only the radiation fields, i.e. fields that decay as $1 /|\boldsymbol{r}-\overline{\boldsymbol{r}}|$ with distance between the source and the observer. It should be noted, that near-field of point-like emitters can contain a wealth of interesting information on emission and re-absorption of electromagnetic energy $[23,24]$, however here we shall forgo this in the interest of simplicity.

The short-cut can be illustrated by the simple case of $n=0$ and $K^{\mu}=q c^{2} \hat{\tau}^{\mu}$ (current density due to pointcharge [9]):

$$
F_{\mu \eta}^{(n=0)}=\frac{\mu_{0}}{2 \pi} \delta_{\mu \eta}^{\gamma \xi} g_{\xi \kappa} \int d \tau K^{\kappa} \nabla_{\gamma} \delta(X \cdot X)
$$

Following Ref. [9] we rewrite $\left(u^{\mu}=d \bar{x}^{\mu} / d \tau\right.$ is the fourvelocity):

$$
\begin{aligned}
\nabla_{\gamma} \delta(X \cdot X) & =\nabla_{\gamma} \delta\left((x-\bar{x})_{\sigma}(x-\bar{x})^{\sigma}\right) \\
& =-\frac{X_{\gamma}}{u \cdot X} \frac{d}{d \tau} \delta(X \cdot X)
\end{aligned}
$$

Substituting and integrating by parts:

$$
\begin{aligned}
F_{\mu \eta}^{(n=0)} & =\frac{\mu_{0}}{2 \pi} \delta_{\mu \eta}^{\gamma \xi} g_{\xi \kappa} \int d \tau\left(-\frac{X_{\gamma}}{u \cdot X}\right) K^{\kappa} \frac{d}{d \tau} \delta(X \cdot X) \\
& =\frac{\mu_{0}}{2 \pi} \delta_{\mu \eta}^{\gamma \xi} g_{\xi \kappa} \int d \tau \frac{d}{d \tau}\left[\left(\frac{X_{\gamma}}{u \cdot X}\right) K^{\kappa}\right] \delta(X \cdot X)
\end{aligned}
$$



[9]:

The one-dimensional delta-function can be re-cast into

$$
\delta(X \cdot X)=\frac{1}{2(u \cdot X)} \delta\left(\tau-\tau_{r e t}\right)
$$

Where $\tau_{r e t}$ is the proper time at which $X^{\sigma}=(r, \boldsymbol{r})^{\sigma}$, i.e. when the observer located at position $\boldsymbol{r}$ away from the point-particle observes the radiation emitted by the point-particle at time $r / c$ earlier. For any function of proper time $f=f(\tau)[9]$ :

$$
\int d \tau \delta(X \cdot X) f(\tau)=\left[\frac{f(\tau)}{2(u \cdot X)}\right]_{\tau=\tau_{r e t}}=\frac{f\left(\tau_{r e t}\right)}{2 r \gamma(c-\hat{\boldsymbol{r}} \cdot \boldsymbol{v})}
$$

Above $\boldsymbol{v}=d \overline{\boldsymbol{r}} / d t$ is the particle velocity in frame $S$ (at time that corresponds to $\left.\tau_{r e t}\right)$, and $\gamma=1 / \sqrt{1-(v / c)^{2}}$ is the Lorentz factor. The key observation about the above equation is that it is already decaying as $1 / r$ with the distance between the point-multipole and the observer. Therefore if one wants the integral above to decay as $1 / r$, $f\left(\tau_{r e t}\right)$ must not decay with $r$ at all, which means that it has to be zeroth order in $X^{\sigma}$ (since $X^{\sigma} \rightarrow r(1, \hat{\boldsymbol{r}})^{\sigma}$ when $\tau \rightarrow \tau_{\text {ret }}$ ).
Returning to Eq. (10), and using $u^{\mu}=d \bar{x} / d \tau$ and $a^{\mu}=$ $d u^{\mu} / d \tau$ :

$$
\begin{aligned}
& \frac{d}{d \tau}\left[\left(\frac{X_{\gamma}}{u \cdot X}\right) K^{\kappa}\right]=\frac{-u_{\gamma}}{u \cdot X} K^{\kappa}- \\
& \quad-\frac{X_{\gamma}}{(u \cdot X)^{2}} K^{\kappa}\left(a \cdot X-c^{2}\right)+\frac{X_{\gamma}}{u \cdot X} \frac{d}{d \tau}\left(K^{\kappa}\right)
\end{aligned}
$$

Above, one can see that all the terms obtained by differentiating $X$ will be of order $1 / X$ and will therefore not contribute to far-field radiation. One can also see that this has to be a general prescription. Function $K^{\cdots}$ does not depend on $X$, and the only way that $X$ appears under the integral is via Eq. (9). However, at that point the whole expression is zeroth order in $X$ ( $X$ appears in the numerator and the denominator), thus any differentiation of $X$ will make the expression negative order in $X$. Therefore the rule for obtaining only the far-field radiation contributions becomes: not to differentiate $X$. We shall denote this by putting a dot above the equals sign, so, for example:

$$
\frac{d}{d \tau}\left[\left(\frac{X_{\gamma}}{u \cdot X}\right) K^{\kappa}\right] \doteq-\frac{X_{\gamma}(a \cdot X)}{(u \cdot X)^{2}} K^{\kappa}+\frac{X_{\gamma}}{u \cdot X} \frac{d}{d \tau}\left(K^{\kappa}\right)
$$

This rule allows to recast Eq. (8) into a form more suitable for evaluation:

$$
\begin{aligned}
& F_{\mu \eta} \doteq \frac{\mu_{0}}{2 \pi} \delta_{\mu \eta}^{\gamma \xi} g_{\xi \kappa} \int d \tau \frac{d}{d \tau}\left[\left(\frac{X_{\gamma}}{u \cdot X}\right) \frac{d}{d \tau}\left[\left(\frac{X_{\alpha_{1}}}{u \cdot X}\right) \ldots \frac{d}{d \tau}\left[\left(\frac{X_{\alpha_{n}}}{u \cdot X}\right) K^{\kappa \alpha_{1} \ldots \alpha_{n}}\right] \ldots\right]\right] \delta(X \cdot X) \\
& F_{\mu \eta} \doteq \frac{\mu_{0}}{4 \pi} \delta_{\mu \eta}^{\gamma \xi} g_{\xi \kappa}\left[\frac{1}{(u \cdot X)} \frac{d}{d \tau}\left[\left(\frac{X_{\gamma}}{u \cdot X}\right) \frac{d}{d \tau}\left[\left(\frac{X_{\alpha_{1}}}{u \cdot X}\right) \ldots \frac{d}{d \tau}\left[\left(\frac{X_{\alpha_{n}}}{u \cdot X}\right) K^{\kappa \alpha_{1} \ldots \alpha_{n}}\right] \ldots\right]\right]\right]_{\tau=\tau_{r e t}}
\end{aligned}
$$

\section{RADIATION FROM UNIFORMLY ACCELERATING POINT-PARTICLE ELECTRIC, TOROIDAL AND ANAPOLE DIPOLES}

In this section we will apply Eq. (12) to find the far-field radiation from point-particle with electric (Sec. IV A), toroidal (Sec. IV B), and anapole (Sec. IV C) dipolar moments.

Before proceeding we shall briefly review the expressions specific to motion of uniformly accelerated pointparticles. The world-line of a point-particle, in the labframe, with uniform acceleration $a$, along the z-axis, is $[25]$ :

$$
\begin{aligned}
& \bar{x}^{\mu}(\tau)=\frac{c^{2}}{a}\left(\sinh \frac{a \tau}{c}, 0,0, \cosh \frac{a \tau}{c}-1\right)^{\mu} \\
& \bar{x}^{\mu}(t)=\left(c t, 0,0, \frac{c^{2}}{a}\left(\sqrt{1+(a t / c)^{2}}-1\right)\right)^{\mu}
\end{aligned}
$$

Above, $\tau$ is the proper time of the accelerating particle and the over-bar above $\bar{x}^{\mu}$ is introduced used to distinguish the position of the accelerating particle from the location of a generic event. The integration constants are chosen so that at time $t=0$, which also corresponds to proper time $\tau=0$, the accelerating particle is passing through the lab-frame origin, and is instantaneously at rest in the lab-frame. The parameter $a=\sqrt{-a^{\mu} a_{\mu}}$ is the magnitude of the four-acceleration $a^{\mu}=d^{2} \bar{x}^{\mu} / d \tau^{2}$.

The worldline of the observer (following Eq. (13)) is shown in Fig. 2. It is convenient to introduce four 


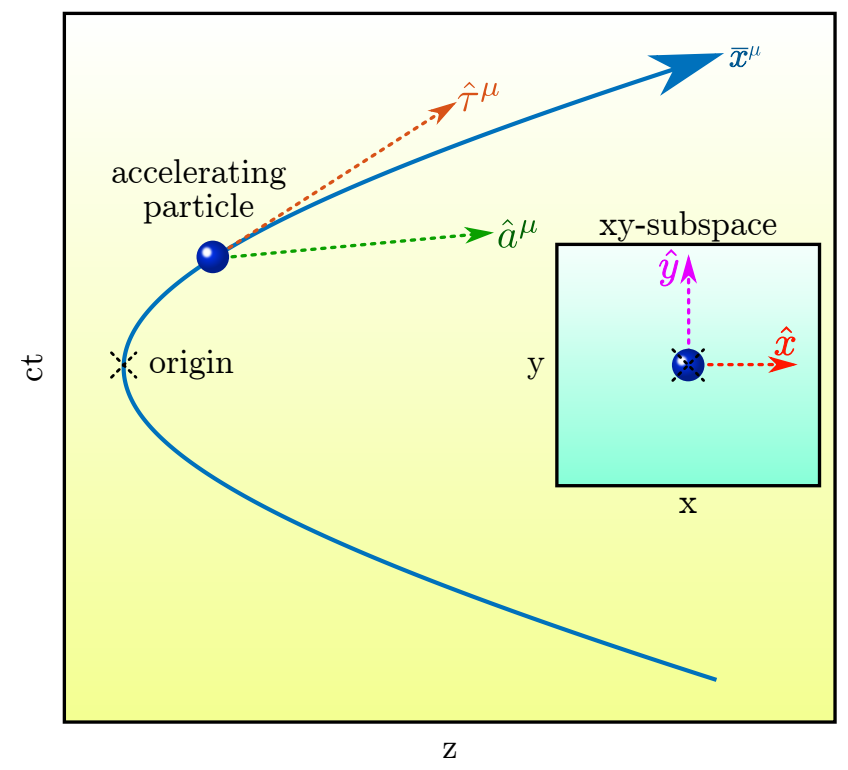

Figure 2. Worldline of the uniformly accelerating particle. Blue curve (hyperbola) shows the path of the uniformly accelerating particle (blue dot). Vectors $\hat{\tau}^{\mu}$ and $\hat{a}^{\mu}$ are orthonormal vectors in the direction of instantaneous four-velocity, and four-acceleration. Together they span $t z-$ subspace. The inset shows two other orthonormal vector $\hat{x}^{\mu}$ and $\hat{y}^{\mu}$ which span the $x y$-subspace. The four vectors form a complete orthonormal set that spans the space-time. Speed of light is denoted by $c$.

four-vectors that form an orthonormal set that spans the spacetime. Vector $\hat{\tau}^{\mu}$ points in the direction tangent to the worldline of the accelerating particle, and is therefore parallel to four-velocity $d \bar{x}^{\mu} / d \tau=u^{\mu}=c \hat{\tau}^{\mu}$ $\left(\hat{\tau} \cdot \hat{\tau}=\hat{\tau}^{\mu} \hat{\tau}_{\mu}=1\right)$, vector $\hat{a}^{\mu}$ is parallel to fouracceleration $a^{\mu}=d u^{\mu} / d \tau=a \hat{a}^{\mu}(\hat{a} \cdot \hat{a}=-1)$, finally vectors $\hat{x}^{\mu}$ and $\hat{y}^{\mu}$ are along the $x$ - and $y$-axis $(\hat{x} \cdot \hat{x}=-1$ and $\hat{y} \cdot \hat{y}=-1)$.

From Eq. (13) it follows:

$$
\begin{aligned}
\frac{d u^{\mu}}{d \tau} & =a^{\mu}=a \hat{a}^{\mu} & \frac{d \hat{a}^{\mu}}{d \tau} & =\frac{a}{c} \hat{\tau}^{\mu} \\
\frac{d \hat{\tau}^{\mu}}{d \tau} & =\frac{a}{c} \hat{a}^{\mu} & \frac{d a^{\mu}}{d \tau} & =\left(\frac{a}{c}\right)^{2} u^{\mu}=\frac{a^{2}}{c} \hat{\tau}^{\mu}
\end{aligned}
$$

Both in case of electric (Eq. (4)) and toroidal (Eq. (5)) dipole we will be working with vectors that are, by definition, always orthogonal to the world-line of the particle, i.e. $p \cdot \hat{\tau}=p^{\mu} \hat{\tau}_{\mu}=0$ for electric dipole four-vector (and the same for $T^{\mu}$ ). Such vectors can be conveniently parametrized by:

$$
p^{\mu}=p^{1} \hat{x}^{\mu}+p^{2} \hat{y}^{\mu}+p^{a} \hat{a}^{\mu}
$$

We shall be interested in proper-time derivatives of such vectors. Introducing $\dot{p}^{\mu} \equiv \frac{d p^{1}}{d \tau} \hat{x}^{\sigma}+\frac{d p^{2}}{d \tau} \hat{y}^{\sigma}+\frac{d p^{a}}{d \tau} \hat{a}^{\sigma}$ (and the corresponding equivalent for $\ddot{p}^{\sigma}$ etc.), one finds:

$$
\begin{aligned}
\frac{d p^{\mu}}{d \tau} & =\dot{p}^{\mu}+p^{a} \frac{d \hat{a}^{\mu}}{d \tau}=\dot{p}^{\mu}+(-p \cdot \hat{a})\left(\frac{a}{c} \hat{\tau}^{\mu}\right) \\
& =\dot{p}^{\mu}-\frac{a}{c}(p \cdot \hat{a}) \hat{\tau}^{\mu}
\end{aligned}
$$

\section{A. Uniformly accelerated electric dipole point-particle}

The suitable current density for electric point-particle with electric dipole is given in Eq. (4). Substituting it into Eq. (12), the electromagnetic tensor for the far-field radiation from a point-particle electric dipole becomes:

$$
\begin{aligned}
F_{\mu \eta}^{(p)} \doteq \frac{\mu_{0} c^{2}}{2 \pi} \delta_{\mu \eta}^{\gamma \xi} \delta_{\sigma \rho}^{\kappa \alpha} g_{\xi \kappa}\left[\frac{1}{2(u \cdot X)} \frac{d}{d \tau}[\right. \\
\left.\left.\left(\frac{X_{\gamma}}{u \cdot X}\right) \frac{d}{d \tau}\left[\left(\frac{X_{\alpha}}{u \cdot X}\right) p^{\sigma} \hat{\tau}^{\rho}\right]\right]\right]_{\tau=\tau_{r e t}}
\end{aligned}
$$

next one applies the relations from the introductory part of Sec. IV to find radiation in the case of uniform acceleration:

$$
\begin{aligned}
& F_{\mu \eta}^{(p)} \doteq \frac{\mu_{0} c^{2}}{4 \pi} \delta_{\mu \eta}^{\gamma \xi} \delta_{\sigma \rho}^{\kappa \alpha} g_{\xi \kappa} {\left[\frac{X_{\alpha} X_{\gamma}}{(u \cdot X)^{3}}[\right.} \\
&\left(\ddot{p}^{\sigma}-3\left(\frac{a \cdot X}{u \cdot X}\right) \dot{p}^{\sigma}+3\left(\frac{a \cdot X}{u \cdot X}\right)^{2} p^{\sigma}\right) \hat{\tau}^{\rho}+ \\
&+\frac{a}{c}\left(2 \dot{p}^{\sigma}-3\left(\frac{a \cdot X}{u \cdot X}\right) p^{\sigma}\right) \hat{a}^{\rho}- \\
&\left.\left.-\left(\frac{a}{c}\right)^{2}(p \cdot \hat{a}) \hat{\tau}^{\sigma} \hat{a}^{\rho}\right]\right]_{\tau=\tau_{r e t}}
\end{aligned}
$$

Above, the anti-symmetric property of Kronecker delta was used implicitly, e.g. $\delta_{\sigma \rho}^{\kappa \alpha} \hat{\tau}^{\sigma} \hat{\tau}^{\rho}=0$. Next, we simplify the expression by assuming that $a \tau / c \rightarrow 0$, i.e. we will assume that during the observation period the speed of the accelerating point-particle is negligible in the labframe. At the same time the particle will be located at the origin in the lab-frame (see Eq. (13) and Fig. 2). One important consequence of this approximation is that electric dipole of the particle in the lab-frame becomes the same as in its (instantaneous) rest-frame, one can therefore talk about a three-dimensional dipole moment vector $\boldsymbol{p}$ without ambiguity. Some of the transformations due to this approximation are (see Eq. (13) and Fig. 2):

$$
\begin{array}{ccc}
\hat{a}^{\mu} \rightarrow \hat{z}^{\mu}=\delta_{3}^{\mu}, & p^{\mu} \rightarrow(0, \boldsymbol{p})^{\mu}, & \hat{\tau}^{\mu} \rightarrow \hat{t}^{\mu}=\delta_{0}^{\mu} \\
u^{\mu} \rightarrow c \delta_{0}^{\mu}, & \tau_{r e t} \rightarrow t-r / c, & (u \cdot X) \rightarrow r c \\
(a \cdot X) \rightarrow-a r \hat{\boldsymbol{r}} . \hat{\boldsymbol{a}} & X^{\mu} \rightarrow r(1, \hat{\boldsymbol{r}})^{\mu}, & (\hat{a} \cdot p) \rightarrow-\hat{\boldsymbol{a}} \cdot \boldsymbol{p}
\end{array}
$$

It is important to note that this approximation can only be applied at the end, once all derivatives have been 
evaluated. This last approximation will be denoted by asterix over the equals sign $(\stackrel{*}{=})$. Next, we evaluate Eq. (15) and use the result to find electric $\left(\left(\boldsymbol{E}^{(p)}\right)^{i}=-c g^{i j} F_{0 j}^{(p)}\right)$ and magnetic fields $\left(\left(\mu_{0} \boldsymbol{H}^{(p)}\right)^{i}=-\epsilon^{i a b} F_{a b}^{(p)} / 2\right)$ :

$$
\begin{aligned}
\boldsymbol{E}^{(p)} \stackrel{*}{=} & \frac{\mu_{0}}{4 \pi r}[\hat{\boldsymbol{r}} \times \hat{\boldsymbol{r}} \times \mathcal{V}- \\
& \left.-\left(\frac{a}{c}\right) \hat{\boldsymbol{r}} \times \hat{\boldsymbol{a}} \times \mathcal{W}-\left(\frac{a}{c}\right)^{2}(\boldsymbol{p} . \hat{\boldsymbol{a}}) \hat{\boldsymbol{r}} \times \hat{\boldsymbol{r}} \times \hat{\boldsymbol{a}}\right] \\
\boldsymbol{H}^{(p)} \stackrel{*}{=} & \frac{1}{\mu_{0} c} \hat{\boldsymbol{r}} \times \boldsymbol{E}^{(p)} \\
\mathcal{V}= & \ddot{\boldsymbol{p}}+3 \frac{a}{c}(\hat{\boldsymbol{a}} . \hat{\boldsymbol{r}}) \dot{\boldsymbol{p}}+3\left(\frac{a}{c}\right)^{2}(\hat{\boldsymbol{a}} . \hat{\boldsymbol{r}})^{2} \boldsymbol{p} \\
\mathcal{W} & =2 \dot{\boldsymbol{p}}+3 \frac{a}{c}(\hat{\boldsymbol{a}} . \hat{\boldsymbol{r}}) \boldsymbol{p} \\
\boldsymbol{p} & =\boldsymbol{p}(t-r / c)
\end{aligned}
$$

Above we have introduced vector fields $\mathcal{V}$ and $\mathcal{W}$ for convenience. Here and for the rest of the text we remove the nested brackets from cross-products, but our convention is to apply them from right to left, i.e. $\boldsymbol{U} \times \boldsymbol{V} \times \boldsymbol{W}=\boldsymbol{U} \times(\boldsymbol{V} \times \boldsymbol{W})$. Both electric and magnetic fields are manifestly transverse, since they can be written as $\hat{\boldsymbol{r}} \times \ldots$ It follows that $\boldsymbol{E}^{(p)} \times \boldsymbol{H}^{(p)}=$ $\frac{1}{\mu_{0} c}\left(\hat{\boldsymbol{r}}\left|\boldsymbol{E}^{(p)}\right|^{2}-\boldsymbol{E}^{(p)}\left(\hat{\boldsymbol{r}} . \boldsymbol{E}^{(p)}\right)\right)=\left(\left|\boldsymbol{E}^{(p)}\right|^{2} / \mu_{0} c\right) \hat{\boldsymbol{r}}$. Thus, as one should expect, the fields result in Poynting vector in positive radial direction, i.e. electromagnetic energy is propagating away from the dipole.

\section{B. Uniformly accelerated toroidal dipole point-particle}

The suitable current density is given in Eq. (5). Substituting it into Eq. (12) results in the following electromagnetic tensor:

$$
F_{\mu \eta}^{(T)} \doteq \frac{\mu_{0} c^{2}}{4 \pi} \delta_{\mu \eta}^{\theta \xi} g_{\xi \kappa} \delta_{\phi \sigma \rho}^{\kappa \nu \alpha} g^{\rho \beta}\left[\frac{1}{u \cdot X} \frac{d}{d \tau}\left[\left(\frac{X_{\theta}}{u \cdot X}\right) \frac{d}{d \tau}\left[\left(\frac{X_{\alpha}}{u \cdot X}\right) \frac{d}{d \tau}\left[\left(\frac{X_{\beta}}{u \cdot X}\right) T^{\phi} \hat{\tau}^{\sigma} \hat{\tau}_{\nu}\right]\right]\right]\right]_{\tau=\tau_{\text {ret }}}
$$

Evaluation of the above expression is significantly more difficult than Eq. (15): there a more derivatives and more terms. The details of the evaluation are therefore given in the App. B, whilst here we give only the final result:

$$
\begin{aligned}
& \boldsymbol{E}^{(T)} \stackrel{*}{=} \frac{\mu_{0}}{4 \pi r c}\left[-\hat{\boldsymbol{r}} \times \hat{\boldsymbol{r}} \times \mathcal{F}+3\left(\frac{a}{c}\right) \hat{\boldsymbol{r}} \times \hat{\boldsymbol{a}} \times \mathcal{Q}+\right. \\
& \left.\quad+3\left(\frac{a}{c}\right)^{2}(\hat{\boldsymbol{a}} \cdot \mathcal{G}) \hat{\boldsymbol{r}} \times \hat{\boldsymbol{r}} \times \hat{\boldsymbol{a}}\right] \\
& \boldsymbol{H}^{(T)} \stackrel{*}{=} \frac{1}{\mu_{0} c} \hat{\boldsymbol{r}} \times \boldsymbol{E}^{(T)}
\end{aligned}
$$

With:

$$
\begin{aligned}
& \mathcal{F}=\dddot{\boldsymbol{T}}+3\left(\frac{a}{c}\right)(\hat{\boldsymbol{a}} \cdot \hat{\boldsymbol{r}}) \ddot{\boldsymbol{T}}+\left(\frac{a}{c}\right)^{2}\left(2+3(\hat{\boldsymbol{a}} . \hat{\boldsymbol{r}})^{2}\right) \dot{\boldsymbol{T}}+ \\
&+3\left(\frac{a}{c}\right)^{3}(\hat{\boldsymbol{a}} . \hat{\boldsymbol{r}}) \boldsymbol{T} \\
& \mathcal{Q}=\ddot{\boldsymbol{T}}+2\left(\frac{a}{c}\right)(\hat{\boldsymbol{a}} . \hat{\boldsymbol{r}}) \dot{\boldsymbol{T}}+\left(\frac{a}{c}\right)^{2}(\hat{\boldsymbol{a}} . \hat{\boldsymbol{r}})^{2} \boldsymbol{T} \\
& \mathcal{G}=\dot{\boldsymbol{T}}+\left(\frac{a}{c}\right)(\hat{\boldsymbol{a}} . \hat{\boldsymbol{r}}) \boldsymbol{T} \\
& \boldsymbol{T}=\boldsymbol{T}(t-r / c)
\end{aligned}
$$

Where $\boldsymbol{T}$ is the toroidal dipole moment of the pointparticle.

\section{Uniformly accelerated anapole point-particle}

Due to linearity of Maxwell's equations the radiation pattern of the accelerated point-particle with anapole moment can be obtained by combining Eqs. (17, 18, 20, 21) with Eq. (3). After some simplification one can show that the far-field, zero-velocity radiation of a point-particle with anapole moment is:

$$
\begin{aligned}
& \boldsymbol{E}^{(N)} \stackrel{*}{=} \frac{\mu_{0}}{4 \pi r c}\left(\frac{a}{c}\right)[\hat{\boldsymbol{r}} \times \hat{\boldsymbol{a}} \times \boldsymbol{\mathcal { R }}+ \\
& \left.+\left(\frac{a}{c}\right) \hat{\boldsymbol{r}} \times \hat{\boldsymbol{r}} \times \hat{\boldsymbol{a}} \times \hat{\boldsymbol{a}} \times \mathcal{S}\right] \\
& \boldsymbol{H}^{(N)} \stackrel{*}{=} \frac{1}{\mu_{0} c} \hat{\boldsymbol{r}} \times \boldsymbol{E}^{(N)}
\end{aligned}
$$

With:

$$
\begin{aligned}
\mathcal{R} & =\ddot{\boldsymbol{N}}+3\left(\frac{a}{c}\right)(\hat{\boldsymbol{a}} . \hat{\boldsymbol{r}}) \dot{\boldsymbol{N}}+3\left(\frac{a}{c}\right)^{2}(\hat{\boldsymbol{a}} \cdot \hat{\boldsymbol{r}})^{2} \boldsymbol{N} \\
\mathcal{S} & =2 \dot{\boldsymbol{N}}+3\left(\frac{a}{c}\right)(\hat{\boldsymbol{a}} . \hat{\boldsymbol{r}}) \boldsymbol{N} \\
\boldsymbol{N} & =\boldsymbol{N}(t-r / c)
\end{aligned}
$$

Where $\boldsymbol{N}$ is the anapole dipole moment as defined in Eq. (3). We note in passing, that whilst in general the anapole particle does not appear as 'pure' anapole to a stationary observer, under zero-velocity approximation, the current density of anapole particle, in Eq. (6), becomes equivalent to 'conventional' anapole, in Eq. $(1,2,3)$. 


\section{DISCUSSION}

In this section we shall develop several properties of the light emitted by accelerated electric, toroidal and anapole dipoles. The radiation patterns of the emitted radiation are discussed in Sec. V A. Section VB discusses how the ellipticity of radiation emitted by accelerated particles can be used to distinguish electric and toroidal dipole radiation. The work is summarized in Sec. V C.

\section{A. Radiation patterns of accelerated point-particle electric, toroidal and anapole dipoles}

Given the expressions for the far-field radiation from accelerated electric, toroidal and anapole dipoles, one can compute their radiation patterns using the Poynting vector:

$$
\boldsymbol{S}(t, \boldsymbol{r})=\boldsymbol{E}(t, \boldsymbol{r}) \times \boldsymbol{H}(t, \boldsymbol{r}) \stackrel{*}{=} \frac{1}{\mu_{0} c}|\boldsymbol{E}(t, \boldsymbol{r})|^{2} \hat{\boldsymbol{r}}
$$

The second equality in the equation above applies only in the far-field, where both electric and magnetic fields are transverse, and where $\boldsymbol{H} \stackrel{*}{=} \hat{\boldsymbol{r}} \times \boldsymbol{E} / \mu_{0} c$. The radiation patterns of all three dipoles are visualized in Fig. 3, which shows time-averaged power-per-solid angle radiated by accelerated point-particles with corresponding dipole moments. For the purposes of visualization, it is assumed that the corresponding dipole moments oscillate along a single axis with angular frequency $\omega$ (e.g. $\boldsymbol{N}=\hat{\boldsymbol{z}} N_{0} \cos \omega t$ for anapole), and that the acceleration of the particle is perpendicular to the dipole moment $(\boldsymbol{N} . \hat{\boldsymbol{a}}=0)$. In this case, the radiation pattern is governed by a single parameter $a / \omega c$ which corresponds to the number of oscillation cycles at frequency $\omega$ it would take for the particle to reach relativistic speed, starting from rest.

At low acceleration, the emission of oscillating electric and toroidal dipoles is indistinguishable, and is in the form of the well-known doughnut-shaped radiation pattern (see Fig. 3 column 2, rows 1,2). The emission of anapole is more interesting. As follows from Eq. (22), the emission of anapole vanishes unless: (a) the anapole is accelerating $(a>0)$; (b) the anapole moment, or its derivatives, are perpendicular to acceleration $(\hat{\boldsymbol{a}} \times \boldsymbol{N} \neq 0$ or $\hat{\boldsymbol{a}} \times \dot{\boldsymbol{N}} \neq 0$ or $\hat{\boldsymbol{a}} \times \ddot{\boldsymbol{N}} \neq 0$ ). The low-acceleration limit of emission from an oscillating anapole point-particle (row 3, column 2 of Fig. 3) has a particularly simple form, with electric field:

$$
\lim _{a \rightarrow 0} \boldsymbol{E}^{(N)} \stackrel{*}{=} \frac{\mu_{0}}{4 \pi r c^{2}}(\hat{\boldsymbol{r}} \times \boldsymbol{a} \times \ddot{\boldsymbol{N}})
$$

which corresponds to emission of a magnetic pointdipole with moment $\boldsymbol{a} \times \boldsymbol{N} / c$.
As intermediate acceleration $(a / \omega c \sim 1)$, the radiation pattern of of all three dipoles becomes significantly more complex (column 3 of Fig. 3). One noteworthy feature is that in this regime the emission patterns of all three dipoles have no zeros, i.e. the dipoles emit in all directions with roughly the same efficiency.

The regime of $a / \omega c \gg 1$ (column 4 in Fig. 3) corresponds both to high acceleration of oscillating dipoles, as well as to emission from static dipoles (where $\omega \rightarrow 0$ ). Here the emission patterns of electric and toroidal dipoles loose any resemblance, whilst the emission of anapole becomes identical to that of a toroidal dipole. Indeed, this agrees with the definition of an anapole given in Eq. (3): static anapole is the toroidal dipole.

\section{B. Distinguishing between electric and toroidal dipoles using ellipticity}

The existence of anapoles, and nonradiating configurations more generally, in electrodynamics is related directly to impossibility to 'invert' the scattering problem, i.e. to uniquely determine the source of electromagnetic radiation based solely on the electromagnetic (far-)field it emits. Here we will consider a method of distinguishing between emission of oscillating electric and toroidal dipole point-particles under acceleration which relies on the ellipticity of the emitted light.

We consider a scenario in which one analyzes the light scattered (re-emitted) by atoms via electric and toroidal dipole excitations. It has already been shown that it is possible to induce both electric [26], and toroidal [27, 28] dipole excitations in atoms. However, as discussed in Sec. I, there is currently no way to experimentally distinguish between them (this is part of the inverse-source problem). The radiation patterns of accelerated dipoles in Fig. 3 suggest that if the atoms in question could be subjected to sufficiently high acceleration, the difference between electric and toroidal dipole excitations would be detectable. We shall now estimate a realistic value for acceleration to which the atoms could be subjected. Let the frequency of the light scattered by the atoms correspond to a free-space wavelength of $\lambda_{0}=1 \mu \mathrm{m}\left(\omega=2 \pi c / \lambda_{0}\right)$. The atoms under consideration could be accelerated in a number of ways including sonoluminescence [29], and laser based methods [30, 31] which can provide accelerations as high as $10^{22} \mathrm{~m} / \mathrm{s}^{2}$. The most serious limitation is that the acceleration should not destroy the atom. The typical forces within the atom are $F_{0}=10^{-7} \mathrm{~N}$ (force on the electron due to the charged nucleus). We shall assume that the accelerated atom has the mass of a carbon atom $\left(m_{C}\right)$, and is accelerated with a force two orders of magnitude smaller than the internal atomic forces so: $a=F_{0} /\left(100 m_{C}\right) \approx 4 \times 10^{16} \mathrm{~m} / \mathrm{s}^{2}$. Therefore $a / \omega c=a \lambda_{0} / 2 \pi c^{2} \approx 10^{-7}$. 


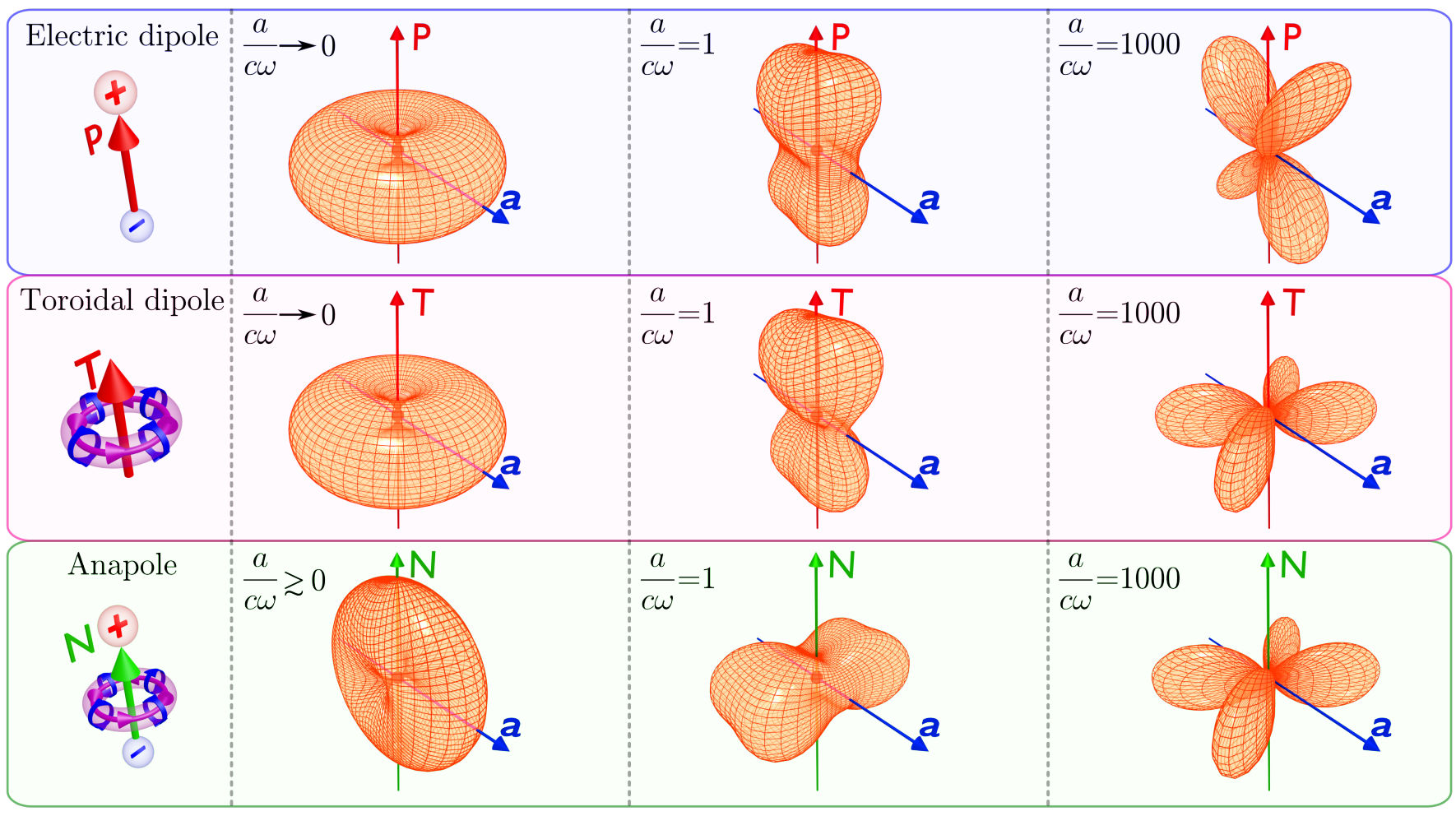

Figure 3. Radiation patterns of accelerating point-particles with electric (top row) and toroidal (middle row) and anapole dipoles moments. The magnitude of acceleration is $a$. In all cases the corresponding dipoles are assumed to be harmonically oscillating along a single axis (e.g. $\boldsymbol{p}=\hat{\boldsymbol{z}} p_{0} \cos (\omega t)$ with $p_{0}=$ const, and the same for $\boldsymbol{T}$ and $\left.\boldsymbol{N}\right)$. The key parameter is $a / \omega c$, where $c$ is the speed of light. This parameter corresponds to how many cycles of oscillation (i.e. periods of $2 \pi / \omega)$ it takes for the accelerating particle to reach relativistic speed (starting from rest). Three cases are considered: the 2nd column shows radiation patterns for the case of low acceleration. For an anapole the acceleration magnitude has to be above zero for non-zero radiation. The 3 rd and 4 th columns show the radiation patterns for the cases of intermediate and high acceleration. The 4 th column also corresponds to the radiation patterns of DC dipoles (i.e. zero-frequency limit $\omega \rightarrow 0, a>0$ ).

The the small effects that will arise in the emission patterns of the atoms subjected to acceleration with $a / \omega c \sim 10^{-7}$ are best detected via interference. In particular, here we shall focus on the ellipticity of the light emitted by accelerated point-particle dipoles. The definition of the ellipticity $\chi$ is illustrated in Fig. 4a. Given the localized electromagnetic source (at the origin), the polarization of the emitted/scattered light can be decomposed entirely into azimuthal (unit vector $\hat{\phi}$ ) and polar (unit vector $\hat{\boldsymbol{\theta}}$ ) components. Treating the former as 'vertical' and the latter as 'horizontal' components, the ellipticity of the emitted light becomes [32]:

$$
\chi=\arcsin \left(\frac{2 \Im\left(\tilde{E}_{\phi}^{*} \tilde{E}_{\theta}\right)}{\left|\tilde{E}_{\theta}\right|^{2}+\left|\tilde{E}_{\phi}\right|^{2}}\right) / 2
$$

Above we have switched to time-harmonic (electric) fields (denoted by . ..) with time-dependence given by $\exp (+i \omega t)$. In what is to follow it shall be assumed that the relevant dipoles are aligned along z-axis and that the acceleration is along $\mathrm{x}$-axis. The tangent of ellipticity angle $\tan \chi$ is the ratio of the semi-minor to semi-major radius of the ellipse traced out by the electric field emitted from the source (see Fig. 4a). The range of ellipticity is $-\pi / 4 \leq \chi \leq \pi / 4$, with two extremes corresponding to right- $(\chi=\pi / 4)$ and left-circular $(\chi=-\pi / 4)$ polarization, and $\chi=0$ corresponding to linear polarization.

At zero acceleration the emission of both electric and toroidal dipoles is linearly polarized (along $\hat{\boldsymbol{\theta}}$ ), the ellipticity is therefore zero. Acceleration leads to elliptical polarization by adding a delayed orthogonally polarized electric field to the main emission pattern. Using Eq. (25) and the time-harmonic versions of Eqs. $(17,20)$, the ellipticity of the radiation from accelerated electric $\left(\chi^{(p)}\right)$ and toroidal $\left(\chi^{(T)}\right)$ dipoles can be shown to be:

$$
\begin{aligned}
\chi^{(p)} & =2 \cot (\theta) \sin (\phi) \cdot \frac{a}{\omega c}+\mathcal{O}\left(\left(\frac{a}{\omega c}\right)^{3}\right) \\
\chi^{(T)} & =3 \cot (\theta) \sin (\phi) \cdot \frac{a}{\omega c}+\mathcal{O}\left(\left(\frac{a}{\omega c}\right)^{3}\right)
\end{aligned}
$$

Thus, for low accelerations $\chi^{(p)} / \chi^{(T)} \approx 2 / 3$. This 
(a)

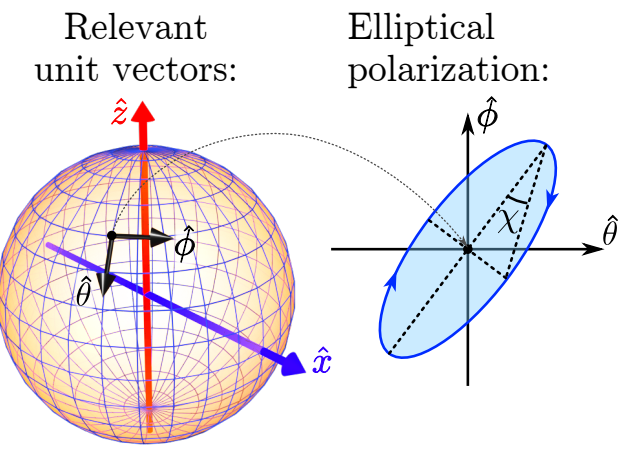

(b)

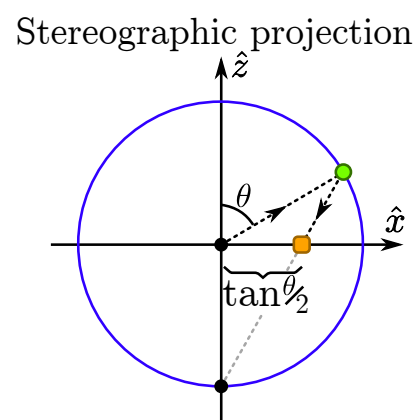

Point $\bigcirc$ is projected ontoo

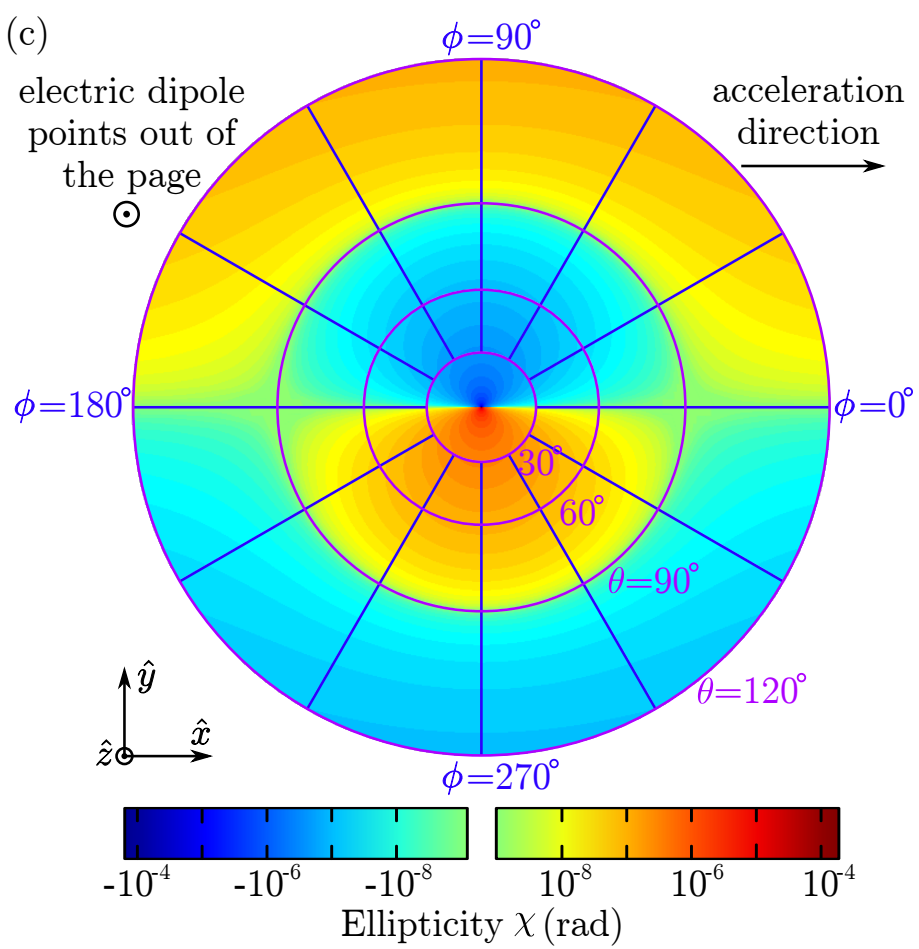

Figure 4. Ellipticity of the light emitted by electric dipole point-particle. (a) Definition of the ellipticity $\chi$. The radiation is emitted by a localized particle at the origin. Since polarization of the emitted radiation is transverse, one can decompose it into azimuthal $(\hat{\boldsymbol{\phi}})$ and polar $(\hat{\boldsymbol{\theta}})$ components. Elliptical polarization corresponds to electric field of the emitted radiation tracing an ellipse in the $\phi \theta$-plane. Ellipticity $(\chi)$ is defined as the angle, the tangent of which is the ratio of the semi-minor and semi-major radii of the polarization ellipse. Positive angle of ellipticity corresponds to electric field rotating clock-wise (as shown in (a)) around the axis of radiation propagation. (b) Definition of the stereographic projection. The projection allows mapping a scalar field, defined on a surface of a unit sphere, onto a flat plane. Here, for simplicity, we show a circle instead of the sphere and a line instead of the plane. A chosen point on the circle (green circle), which corresponds to angle $\theta$ is projected onto x-axis by plotting a straight line from the south-pole of the circle to the chosen point. The point at which the straight line intersects the x-axis corresponds to the position of the projected point (orange square). Formally, all points on the surface of a sphere, parametrized by $(\theta, \phi)$, are projected onto points on a plane with Cartesian coordinates $\left(\tan \frac{\theta}{2} \cos \phi, \tan \frac{\theta}{2} \sin \phi\right.$ ). (c) Colormap of the ellipticity of the radiation from the accelerated electric dipole point-particle. The electric dipole moment of the particle is $\boldsymbol{p}=p_{0} \cos (\omega t) \hat{\boldsymbol{z}}$, whilst the normalized acceleration is $a / \omega c=10^{-7}$ in the $\hat{\boldsymbol{x}}$-direction. The ellipticity is plotted in a stereographic projection (see (b)) with polar angle ranging from $\theta=0^{\circ}$ (north-pole) to $\theta=120^{\circ}$. The blue color on the colormap corresponds to negative ellipticity, and is clipped for $\chi>-10^{-9} \mathrm{rad}$. The red/orange color corresponds to positive ellipticity and is clipped for $\chi<10^{-9} \mathrm{rad}$.

is a remarkably simple result considering the complexity of the underlying radiation patterns. Ellipticity can be visualized by mapping it onto the unit-sphere and then using stereographic projection to project a portion of the sphere onto the plane. Stereographic projection is illustrated in Fig. 4b. The ellipticity of the radiation emitted by accelerated electric dipole, with normalized acceleration $a / \omega c=10^{-7}$, is shown in Fig. 4c. Whilst $\chi$ is low at the equatorial plane $\left(\theta=90^{\circ}\right)$ where dipole emission is strongest (see Fig. 3), it can be quite high close to the poles, for example at $\theta=13^{\circ}$ and $\phi=90^{\circ}$ (emitted power 20 times less than at equator) $\chi=10^{-6} \mathrm{rad}$. Equations $(26,27)$ suggest that even $10 \%$ sensitivity would be enough to distinguish between electric and toroidal dipoles, i.e. the required ellipticity sensitivity is $\Delta \chi \leq 10^{-7} \mathrm{rad}$. Since ellipticity sensitivity of
$\Delta \chi \leq 10^{-8} \mathrm{rad}[33,34]$ and even $\Delta \chi \leq 10^{-10} \mathrm{rad}$ [35], has already been experimentally demonstrated, we argue that difference between electric and toroidal dipole excitations in atoms should be observable with modern technology.

\section{Conclusion}

In conclusion, we have demonstrated that anapoles, elementary non-radiating configurations, start radiating when accelerated. The consequence of this loop-hole in the inverse-source problem is that one can identify the otherwise inaccessible information on the composition of a source of radiation solely from its emission, provided the source can be accelerated. Further development of 
the acceleration technique presented in this work, to incorporate higher order multipoles, may provide access to even more information on the composition of distant accelerated sources.

In the process of our analysis we have, for the first time, derived expressions for the radiation from an accelerated toroidal and anapole dipoles, and explored polarization properties of the light emitted by accelerated electric and toroidal dipoles. In particular, we have demonstrated that ellipticity of the light emitted by small accelerated particles, such as atoms, can be used to determine whether the emission is due to electric or toroidal dipole transitions. Our work suggests, that there are practical ways to circumvent the inverse-source problem. There are implications for all branches of science where light is used for characterization, from astronomy to biology and high-energy physics.

The author acknowledges financial support from the UK Engineering and Physical Sciences Research Council (Grant No. EP/M009122/1) . Author also gratefully acknowledges helpful discussions with Prof N. I. Zheludev (University of Southampton, UK) and Dr V. A. Fedotov (University of Southampton, UK).

[1] A. J. Devaney and E. Wolf, "Radiating and nonradiating classical current distributions and the fields they generate," Phys. Rev. D, vol. 8, p. 1044, 1973.

[2] N. Bleistein and J. K. Cohen, "Nonuniqueness in the inverse source problem in acoustics and electromagnetics," J. Math. Phys., vol. 18, no. 2, pp. 194-201, 1977.

[3] W. R. Stone, "A review and examination of results on uniqueness in inverse problems," Radio Sci., vol. 22, no. 6, pp. 1026-1030, 1987.

[4] A. J. Devaney, Mathematical Foundations of Imaging, Tomography and Wavefield Inversion. Cambridge University Press, 2012.

[5] L. Novotny and B. Hecht, Principles of Nano-Optics, 2nd ed. Cambridge University Press, 2012.

[6] Ya. B. Zel'dovich, "Electromagnetic interaction with parity violation," Sov. Phys. JETP, vol. 33, p. 1531, 1957.

[7] G. N. Afanasiev and Yu. P. Stepanovsky, "The electromagnetic field of elementary time-dependent toroidal sources," J. Appl. Phys. A, vol. 28, p. 4565, 1995.

[8] N. Papasimakis, V. Fedotov, V. Savinov, T. A. Raybould, and N. I. Zheludev, "Electromagnetic toroidal excitations in matter and free space," Nature Mater., vol. 15, p. 263, 2016.

[9] J. D. Jackson, Classical Electrodynamics. Wiley, New York ed. 3, 1999.

[10] V. A. Fedotov, A. V. Rogacheva, V. Savinov, D. P. Tsai, and N. I. Zheludev, "Resonant transparency and nontrivial non-radiating excitations in toroidal metamaterials," Sci. Rep., vol. 3, p. 2967, 2013.

[11] A. E. Miroshnichenko et al., "Nonradiating anapole modes in dielectric nanoparticles," Nat. Comm., vol. 6, p. 8069, 2015.

[12] N. A. Nemkov, I. V. Stenishchev, and A. A. Basharin,
"Nontrivial nonradiating all-dielectric anapole," Sci. Rep., vol. 7, no. 1, pp. 1064-, 2017.

[13] T. Shibanuma, G. Grinblat, P. Albella, and S. A. Maier, "Efficient third harmonic generation from metaldielectric hybrid nanoantennas," Nano Lett., vol. 17, no. 4, pp. 2647-2651, 2017, pMID: 28288274.

[14] A. A. Basharin, V. Chuguevsky, N. Volsky, M. Kafesaki, and E. N. Economou, "Extremely high Q-factor metamaterials due to anapole excitation," Phys. Rev. B, vol. 95, p. 035104, Jan 2017.

[15] G. Grinblat et al., "Efficient third harmonic generation and nonlinear subwavelength imaging at a higher-order anapole mode in a single germanium nanodisk," $A C S$ Nano, vol. 11, no. 1, pp. 953-960, 2017, pMID: 27977932.

[16] A. J. Devaney and E. Wolf, "Multipole expansions and plane wave representations of the electromagnetic field," J. Math. Phys., vol. 15, p. 234, 1974.

[17] N. A. Nemkov, A. A. Basharin, and V. A. Fedotov, "Nonradiating sources, dynamic anapole, and AharonovBohm effect," Phys. Rev. B, vol. 95, p. 165134, Apr 2017.

[18] J. R. Ellis, "The fields of an arbitrarily moving dipole," Proc. Camb. Phil. Soc., vol. 59, p. 759, 1963.

[19] G. N. Ward, "The electromagnetic fields of moving dipoles," Proc. Camb. Phil. Soc., vol. 61, p. 547, 1965.

[20] J. R. Ellis, "Electromagnetic fields of moving dipoles and multipoles," J. Math. Phys., vol. 7, p. 1185, 1966.

[21] J. J. Monaghan, "The Heaviside-Feynman expression for the fields of an accelerated dipole," J. Phys. A: Gen. Phys., vol. 1, p. 112, 1968.

[22] D. Lovelock and H. Rund, Tensors, Differential Forms and Variational Principles. Dover Publications, 1989.

[23] L. Mandel, "Energy flow from an atomic dipole in classical electrodynamics," J. Opt. Soc. Am., vol. 62, no. 8, pp. 1011-1012, Aug 1972.

[24] E. Bossy and R. Carminati, "Time-domain radiation and absorption by subwavelength sources," EPL (Europhysics Letters), vol. 97, no. 3, p. 34001, 2012.

[25] C. W. Misner, K. S. Thorne, and J. A. Wheeler, Gravitation. W. H. Freeman and Company, 1973.

[26] C. K. Au, "Dynamic multipole polarizability of hydrogen," J. Phys. B: Atom. Molec. Phys., vol. 11, p. 2781, 1978.

[27] A. Costescu and E. E. Radescu, "Dynamic toroid polarizability of atomic hydrogen," Ann. Phys., vol. 209, p. 13, 1991.

[28] R. R. Lewis and A. Góngora, "Stark-Induced anapole magnetic fields in $\mathrm{n}=2$ hydrogen," J. Phys. B: At. Mol. Opt. Phys., vol. 31, p. 3565, 1998.

[29] K. R. Weninger, B. P. Barber, and S. J. Putterman, "Pulsed Mie scattering measurements of the collapse of a sonoluminescing bubble," Phys. Rev. Lett., vol. 78, p. 1799, 1997.

[30] G. A. Mourou, T. Tajima, and S. V. Bulanov, "Optics in the relativistic regime," Rev. Mod. Phys., vol. 78, p. 309, 2006.

[31] C. Maher-McWilliams, P. Douglas and P. F. Barker, "Laser-driven acceleration of neutral particles," Nature Photon., vol. 6, p. 386, 2012.

[32] D. S. Kliger, J. W. Lewis, and C. E. Randall, Polarized Light in Optics and Spectroscopy. Academic Press, 1990.

[33] S. Carusotto, E. Iacopini, and E. Polacco, "Measurement of the magnetic birefringence of noble gases," J. Opt. Soc. Am. B, vol. 1, p. 635, 1984.

[34] R. Cameron, G. Cantatore, A. C. Melissinos, J. Rogers, 
Y. Semertzidis, H. Halama, A. Prodell, F. A. Nezrick, C. Rizzo, and E. Zavattini, "Measurement of the magnetic birefringence of neon gas," J. Opt. Soc. Am., vol. 8, p. 520, 1991.

[35] K. Muroo, N. Ninomiya, M. Yoshino, and Y. Takubo, "Measurement of the Cotto - Mouton constants of noble atoms," J. Opt. Soc. Am. B, vol. 20, p. 2249, 2003.

[36] J. A. Heras, "Electric and magnetic fields of a toroidal dipole in arbitrary motion," Phys. Lett. A, vol. 249, p. 1, 1998. 


\section{APPENDIX: LIGHT EMISSION BY ACCELERATED ELECTRIC, TOROIDAL AND ANAPOLE DIPOLAR SOURCES}

\section{A. Basic conventions/definitions}

In order to keep the paper self-contained the basic conventions that will be used to handle the kinematics of the uniformly accelerated particles will be given here.

In all cases we will be working in flat space-time and standard diagonal metric $g_{\alpha \beta}=\operatorname{diag}(1,-1,-1,-1)$, thus the square of separation between the two events that differ by time $d t$ and spatial position $d \boldsymbol{r}=\hat{\boldsymbol{x}} d x+\hat{\boldsymbol{y}} d y+$ $\hat{z} d z$ is:

$$
d s^{2}=c^{2} d t^{2}-d \boldsymbol{r} . d \boldsymbol{r}=c^{2} d t^{2}-d x^{2}-d y^{2}-d z^{2}
$$

The contraction of two four-vectors will be denoted as:

$$
\begin{aligned}
a \cdot b & =a_{\mu} b^{\mu}=a^{\mu} b_{\mu}=g_{\mu \nu} a^{\mu} b^{\nu}= \\
& =a^{0} b^{0}-a^{1} b^{1}-a^{2} b^{2}-a^{3} b^{3}
\end{aligned}
$$

Greek indices run through $\{0,1,2,3\}=\{t, x, y, z\}$, and repeated index implies summation unless otherwise stated.

The three-vectors will be denoted by bold-face font. The contraction between two three-vectors is denotes as (no summation implied):

$$
\boldsymbol{a} . \boldsymbol{b}=a^{x} b^{x}+a^{y} b^{y}+a^{z} b^{z}=a^{1} b^{1}+a^{2} b^{2}+a^{3} b^{3}
$$

The partial derivative with respect to coordinate $x^{\alpha}$ is denoted as $\partial_{\alpha}=\partial / \partial x^{\alpha}$. The covariant partial derivative along the direction of changing $x^{\alpha}$, based on Levi-Civita connection, is denoted as $\nabla_{\alpha}$.

We will be using the standard Kronecker delta tensor $[22]:$

$$
\delta_{\beta}^{\alpha}=\frac{\partial x^{\alpha}}{\partial x^{\beta}}, \quad \delta_{\beta}^{\alpha}=g^{\alpha \mu} g_{\mu \beta}, \quad \delta_{\beta}^{\alpha}= \begin{cases}1, & \alpha=\beta \\ 0, & \text { otherwise }\end{cases}
$$

Where $g^{\alpha \beta}$ is the inverse metric. The generalized Kronecker delta tensor is defined through the determinant $[22]:$

$$
\delta_{\beta_{1} \ldots \beta_{r}}^{\alpha_{1} \ldots \alpha_{r}}=\left|\begin{array}{ccc}
\delta_{\beta_{1}}^{\alpha_{1}} & \ldots & \delta_{\beta_{r}}^{\alpha_{1}} \\
\vdots & \ddots & \vdots \\
\delta_{\beta_{1}}^{\alpha_{r}} & \ldots & \delta_{\beta_{r}}^{\alpha_{r}}
\end{array}\right|
$$

We will also be using Levi-Civita relative tensors [22]:

$$
\begin{gathered}
\epsilon^{\alpha \beta \gamma \kappa}=\delta_{0123}^{\alpha \beta \gamma \kappa}, \quad \epsilon_{\alpha \beta \gamma \kappa}=\delta_{\alpha \beta \gamma \kappa}^{0123} \\
\delta_{\phi \eta \mu \nu}^{\alpha \beta \gamma \kappa}=\epsilon^{\alpha \beta \gamma \kappa} \epsilon_{\phi \eta \mu \nu}
\end{gathered}
$$

B. Far-field radiation from uniformly accelerating toroidal dipole

Here we evaluate the electromagnetic tensor for the accelerated toroidal dipole point-particle. The starting point is Eq. (19). In the first stage we define $Q_{\nu}^{\phi \sigma}=$ $T^{\phi} \hat{\tau}^{\sigma} \hat{\tau}_{\nu}$ and differentiate the $1 /(u \cdot X)$ terms:

$$
\begin{aligned}
F_{\mu \eta}^{(T)} \doteq & \frac{\mu_{0} c^{2}}{4 \pi} \delta_{\mu \eta}^{\theta \xi} g_{\xi \kappa} \delta_{\phi \sigma \rho}^{\kappa \nu \alpha} g^{\rho \beta}\left[\frac{1}{u \cdot X} \frac{d}{d \tau}\left[\left(\frac{X_{\theta}}{u \cdot X}\right) \frac{d}{d \tau}\left[\left(\frac{X_{\alpha}}{u \cdot X}\right) \frac{d}{d \tau}\left[\left(\frac{X_{\beta}}{u \cdot X}\right) T^{\phi} \hat{\tau}^{\sigma} \hat{\tau}_{\nu}\right]\right]\right]\right]_{\tau=\tau_{\text {ret }}} \\
\doteq & \frac{\mu_{0} c^{2}}{4 \pi} \delta_{\mu \eta}^{\theta \xi} g_{\xi \kappa} \delta_{\phi \sigma \rho}^{\kappa \nu \alpha} g^{\rho \beta}\left[\frac { X _ { \theta } X _ { \alpha } X _ { \beta } } { ( u \cdot X ) ^ { 4 } } \left(\frac{d^{3} Q_{\nu}^{\phi \sigma}}{d \tau^{3}}+\left(-6 \frac{a \cdot X}{u \cdot X}\right) \frac{d^{2} Q_{\nu}^{\phi \sigma}}{d \tau^{2}}+\right.\right. \\
& \left.\left.+\left(15\left(\frac{a \cdot X}{u \cdot X}\right)^{2}-4\left(\frac{a}{c}\right)^{2}\right) \frac{d Q_{\nu}^{\phi \sigma}}{d \tau}+\left(9\left(\frac{a}{c}\right)^{2}\left(\frac{a \cdot X}{u \cdot X}\right)-15\left(\frac{a \cdot X}{u \cdot X}\right)^{3}\right) Q_{\nu}^{\phi \sigma}\right)\right]_{\tau=\tau_{\text {ret }}}
\end{aligned}
$$

Next, one evaluates the derivatives: 


$$
\begin{aligned}
\frac{d Q_{\nu}^{\phi \sigma}}{d \tau}= & \left(\dot{T}^{\phi}\right) \hat{\tau}^{\sigma} \hat{\tau}_{\nu}+\left(\frac{a}{c} T^{\phi}\right) \hat{a}^{\sigma} \hat{\tau}_{\nu}+\left(\frac{a}{c} T^{\phi}\right) \hat{\tau}^{\sigma} \hat{a}_{\nu} \\
\frac{d^{2} Q_{\nu}^{\phi \sigma}}{d \tau^{2}}= & \left(\ddot{T}^{\phi}+2\left(\frac{a}{c}\right)^{2} T^{\phi}\right) \hat{\tau}^{\sigma} \hat{\tau}_{\nu}+\left(2\left(\frac{a}{c}\right) \dot{T}^{\phi}\right) \hat{a}^{\sigma} \hat{\tau}_{\nu}+\left(2\left(\frac{a}{c}\right) \dot{T}^{\phi}\right) \hat{\tau}^{\sigma} \hat{a}_{\nu}+\left(2\left(\frac{a}{c}\right)^{2} T^{\phi}\right) \hat{a}^{\sigma} \hat{a}_{\nu}+ \\
& +\left(-\left(\frac{a}{c}\right)^{2}(\hat{a} \cdot T)\right) \hat{\tau}^{\phi} \hat{a}^{\sigma} \hat{\tau}_{\nu} \\
\frac{d^{3} Q_{\nu}^{\phi \sigma}}{d \tau^{3}}= & \left(\dddot{T}^{\phi}+6\left(\frac{a}{c}\right)^{2} \dot{T}^{\phi}\right) \hat{\tau}^{\sigma} \hat{\tau}_{\nu}+\left(3\left(\frac{a}{c}\right) \ddot{T}^{\phi}+4\left(\frac{a}{c}\right)^{3} T^{\phi}\right) \hat{a}^{\sigma} \hat{\tau}_{\nu}+\left(3\left(\frac{a}{c}\right) \ddot{T}^{\phi}+4\left(\frac{a}{c}\right)^{3} T^{\phi}\right) \hat{\tau}^{\sigma} \hat{a}_{\nu} \\
& +\left(6\left(\frac{a}{c}\right)^{2} \dot{T}^{\phi}\right) \hat{a}^{\sigma} \hat{a}_{\nu}+\left(-3\left(\frac{a}{c}\right)^{2}(\hat{a} \cdot \dot{T})\right) \hat{\tau}^{\phi} \hat{a}^{\sigma} \hat{\tau}_{\nu}+\left(-3\left(\frac{a}{c}\right)^{3}(\hat{a} \cdot T)\right) \hat{\tau}^{\phi} \hat{a}^{\sigma} \hat{a}_{\nu}
\end{aligned}
$$

Above we have used the rule $d T^{\phi} / d \tau=\dot{T}^{\phi}-$ $\frac{a}{c}(\hat{a} \cdot T) \hat{\tau}^{\phi}$, where $\dot{T}^{\phi}=\frac{d T^{1}}{d \tau} \hat{x}^{\phi}+\frac{d T^{2}}{d \tau} \hat{y}^{\phi}+\frac{d T^{a}}{d \tau} \hat{a}^{\phi}$, and the second term in the derivative arises due to $d \hat{a}^{\phi} / d \tau=$ $(a / c) \hat{\tau}^{\phi}$. However, in the presence of $\delta_{\phi \sigma \rho}^{\mu \nu \alpha} \hat{\tau}^{\sigma}$, this rule can be simplified to $\delta_{\phi \sigma \rho}^{\mu \nu \alpha}\left(d T^{\phi} / d \tau\right) \hat{\tau}^{\sigma}=\delta_{\phi \sigma \rho}^{\mu \nu \alpha} \dot{T}^{\phi} \hat{\tau}^{\sigma}$ because of the anti-symmetry of $\delta_{\phi \sigma \rho}^{\mu \nu \alpha}$. Combining Eq. (B1) and Eq. (B2):

$$
\begin{aligned}
F_{\mu \eta}^{(T)} \doteq & \frac{\mu_{0} c^{2}}{4 \pi} \delta_{\mu \eta}^{\theta \xi} g_{\xi \kappa} \delta_{\phi \sigma \rho}^{\kappa \nu \alpha} g^{\rho \beta}\left[\frac{X_{\theta} X_{\alpha} X_{\beta}}{(u \cdot X)^{4}}\{\right. \\
& \left(\dddot{T}^{\phi}-6\left(\frac{a \cdot X}{u \cdot X}\right) \ddot{T}^{\phi}+\left(2\left(\frac{a}{c}\right)^{2}+15\left(\frac{a \cdot X}{u \cdot X}\right)^{2}\right) \dot{T}^{\phi}-\left(3\left(\frac{a}{c}\right)^{2}\left(\frac{a \cdot X}{u \cdot X}\right)+15\left(\frac{a \cdot X}{u \cdot X}\right)^{3}\right) T^{\phi}\right) \hat{\tau}^{\sigma} \hat{\tau}_{\nu}+ \\
& +3\left(\frac{a}{c}\right)\left(\ddot{T}^{\phi}-4\left(\frac{a \cdot X}{u \cdot X}\right) \dot{T}^{\phi}+5\left(\frac{a \cdot X}{u \cdot X}\right)^{2} T^{\phi}\right)\left(\hat{a}^{\sigma} \hat{\tau}_{\nu}+\hat{\tau}^{\sigma} \hat{a}_{\nu}\right)+6\left(\frac{a}{c}\right)^{2}\left(\dot{T}^{\phi}-2\left(\frac{a \cdot X}{u \cdot X}\right) T^{\phi}\right) \hat{a}^{\sigma} \hat{a}_{\nu}- \\
& \left.\left.-3\left(\frac{a}{c}\right)^{2}\left((\hat{a} \cdot \dot{T})-2\left(\frac{a \cdot X}{u \cdot X}\right)(\hat{a} \cdot T)\right) \hat{\tau}^{\phi} \hat{a}^{\sigma} \hat{\tau}_{\nu}-3\left(\frac{a}{c}\right)^{3}(\hat{a} \cdot T) \hat{\tau}^{\phi} \hat{a}^{\sigma} \hat{a}_{\nu}\right\}\right]
\end{aligned}
$$

As with electric dipole, we approximate the above equation for the case when speed of the toroidal dipole, in the lab-frame, is insignificantly small. The necessary transformations are in Eq. (16) and:

$$
\begin{aligned}
T^{\mu} \rightarrow(0, \boldsymbol{T})^{\mu} & \hat{\tau}_{\mu} \rightarrow \delta_{\mu}^{0} \\
\hat{a} \cdot T \rightarrow-\hat{\boldsymbol{a}} \cdot \boldsymbol{T} & \hat{a}_{\mu} \rightarrow-\delta_{\mu}^{3}
\end{aligned}
$$

The electromagnetic tensor of the point-particle with toroidal dipole, that is momentarily at rest in the lab frame then becomes: 


$$
\begin{aligned}
F_{\mu \eta}^{(T)} \stackrel{*}{=} & \frac{\mu_{0}}{4 \pi r c^{2}} \delta_{\mu \eta}^{\theta \xi}(1, \hat{\boldsymbol{r}})_{\theta} g_{\xi \kappa} \delta_{\phi \sigma \beta}^{\kappa \nu \alpha}(1, \hat{\boldsymbol{r}})_{\alpha}(1, \hat{\boldsymbol{r}})^{\beta}\{ \\
& (0, \mathcal{K})^{\phi} \delta_{0}^{\sigma} \delta_{\nu}^{0}+3\left(\frac{a}{c}\right)(0, \mathcal{L})^{\phi}\left(\delta_{3}^{\sigma} \delta_{\nu}^{0}-\delta_{0}^{\sigma} \delta_{\nu}^{3}\right)-6\left(\frac{a}{c}\right)^{2}(0, \mathcal{M})^{\phi} \delta_{3}^{\sigma} \delta_{\nu}^{3}+3\left(\frac{a}{c}\right)^{2}(\hat{\boldsymbol{a}} . \mathcal{M}) \delta_{0}^{\phi} \delta_{3}^{\sigma} \delta_{\nu}^{0}- \\
& \left.-3\left(\frac{a}{c}\right)^{3}(\hat{\boldsymbol{a}} . \boldsymbol{T}) \delta_{0}^{\phi} \delta_{3}^{\sigma} \delta_{\nu}^{3}\right\} \\
\mathcal{K}= & \dddot{\boldsymbol{T}}+6\left(\frac{a}{c}\right)(\hat{\boldsymbol{a}} . \hat{\boldsymbol{r}}) \ddot{\boldsymbol{T}}+\left(\frac{a}{c}\right)^{2}\left(2+15(\hat{\boldsymbol{a}} . \hat{\boldsymbol{r}})^{2}\right) \dot{\boldsymbol{T}}+3\left(\frac{a}{c}\right)^{3}(\hat{\boldsymbol{a}} . \hat{\boldsymbol{r}})\left(1+5(\hat{\boldsymbol{a}} . \hat{\boldsymbol{r}})^{2}\right) \boldsymbol{T} \\
\mathcal{L}= & \ddot{\boldsymbol{T}}+4\left(\frac{a}{c}\right)(\hat{\boldsymbol{a}} . \hat{\boldsymbol{r}}) \dot{\boldsymbol{T}}+5\left(\frac{a}{c}\right)^{2}(\hat{\boldsymbol{a}} . \hat{\boldsymbol{r}})^{2} \boldsymbol{T} \\
\mathcal{M}= & \dot{\boldsymbol{T}}+2\left(\frac{a}{c}\right)(\hat{\boldsymbol{a}} . \hat{\boldsymbol{r}}) \boldsymbol{T}
\end{aligned}
$$

Above the, vectors $\mathcal{K}, \mathcal{L}$, and $\mathcal{M}$ were introduced to simplify and shorten expression. Next, we find the elec-

tric $\left((\boldsymbol{E})^{i}=-g^{i s} c F_{0 s}\right)$ and magnetic fields $\left(\left(\mu_{0} \boldsymbol{H}\right)^{i}=\right.$ $\left.-\epsilon^{i a b} F_{a b} / 2\right)$ :

$$
\begin{aligned}
& \boldsymbol{E}^{(T)} \stackrel{*}{=} \frac{\mu_{0}}{4 \pi r c}\left[-\hat{\boldsymbol{r}} \times \hat{\boldsymbol{r}} \times \mathcal{K}+3\left(\frac{a}{c}\right) \hat{\boldsymbol{r}} \times \hat{\boldsymbol{a}} \times \mathcal{L}+3\left(\frac{a}{c}\right)(\hat{\boldsymbol{a}} . \hat{\boldsymbol{r}}) \hat{\boldsymbol{r}} \times \hat{\boldsymbol{r}} \times \mathcal{L}-6\left(\frac{a}{c}\right)^{2}(\hat{\boldsymbol{a}} . \hat{\boldsymbol{r}}) \hat{\boldsymbol{r}} \times \hat{\boldsymbol{a}} \times \mathcal{M}+\right. \\
& \left.+3\left(\frac{a}{c}\right)^{2}(\hat{\boldsymbol{a}} . \mathcal{M}) \hat{\boldsymbol{r}} \times \hat{\boldsymbol{r}} \times \hat{\boldsymbol{a}}-3\left(\frac{a}{c}\right)^{3}(\hat{\boldsymbol{a}} . \hat{\boldsymbol{r}})(\hat{\boldsymbol{a}} . \boldsymbol{T}) \hat{\boldsymbol{r}} \times \hat{\boldsymbol{r}} \times \hat{\boldsymbol{a}}\right]_{\boldsymbol{T}=\boldsymbol{T}(t-r / c)} \\
& \boldsymbol{H}^{(T)} \stackrel{*}{=} \frac{1}{4 \pi r c^{2}}\left[\hat{\boldsymbol{r}} \times \mathcal{K}+3\left(\frac{a}{c}\right) \hat{\boldsymbol{r}} \times \hat{\boldsymbol{r}} \times \hat{\boldsymbol{a}} \times \mathcal{L}-3\left(\frac{a}{c}\right)(\hat{\boldsymbol{a}} . \hat{\boldsymbol{r}}) \hat{\boldsymbol{r}} \times \mathcal{L}-6\left(\frac{a}{c}\right)^{2}(\hat{\boldsymbol{a}} . \hat{\boldsymbol{r}}) \hat{\boldsymbol{r}} \times \hat{\boldsymbol{r}} \times \hat{\boldsymbol{a}} \times \mathcal{M}-\right. \\
& \left.-3\left(\frac{a}{c}\right)^{2}(\hat{\boldsymbol{a}} . \mathcal{M}) \hat{\boldsymbol{r}} \times \hat{\boldsymbol{a}}+3\left(\frac{a}{c}\right)^{3}(\hat{\boldsymbol{a}} \cdot \hat{\boldsymbol{r}})(\hat{\boldsymbol{a}} . \boldsymbol{T}) \hat{\boldsymbol{r}} \times \hat{\boldsymbol{a}}\right]_{\boldsymbol{T}=\boldsymbol{T}(t-r / c)}
\end{aligned}
$$

The expressions above can be recombined and shortened. To this end we introduce the following vectors:

$$
\begin{aligned}
& \mathcal{F}= \dddot{\boldsymbol{T}}+3\left(\frac{a}{c}\right)(\hat{\boldsymbol{a}} . \hat{\boldsymbol{r}}) \ddot{\boldsymbol{T}}+\left(\frac{a}{c}\right)^{2}\left(2+3(\hat{\boldsymbol{a}} . \hat{\boldsymbol{r}})^{2}\right) \dot{\boldsymbol{T}}+ \\
&+3\left(\frac{a}{c}\right)^{3}(\hat{\boldsymbol{a}} . \hat{\boldsymbol{r}}) \boldsymbol{T} \\
& \mathcal{Q}=\ddot{\boldsymbol{T}}+2\left(\frac{a}{c}\right)(\hat{\boldsymbol{a}} \cdot \hat{\boldsymbol{r}}) \dot{\boldsymbol{T}}+\left(\frac{a}{c}\right)^{2}(\hat{\boldsymbol{a}} . \hat{\boldsymbol{r}})^{2} \boldsymbol{T} \\
& \mathcal{G}=\dot{\boldsymbol{T}}+\left(\frac{a}{c}\right)(\hat{\boldsymbol{a}} . \hat{\boldsymbol{r}}) \boldsymbol{T}
\end{aligned}
$$

$$
\begin{array}{r}
\boldsymbol{E}^{(T) \stackrel{*}{=}} \frac{\mu_{0}}{4 \pi r c}\left[-\hat{\boldsymbol{r}} \times \hat{\boldsymbol{r}} \times \mathcal{F}+3\left(\frac{a}{c}\right) \hat{\boldsymbol{r}} \times \hat{\boldsymbol{a}} \times \mathcal{Q}+\right. \\
\left.+3\left(\frac{a}{c}\right)^{2}(\hat{\boldsymbol{a}} \cdot \mathcal{G}) \hat{\boldsymbol{r}} \times \hat{\boldsymbol{r}} \times \hat{\boldsymbol{a}}\right]_{\boldsymbol{T}=\boldsymbol{T}(t-r / c)} \\
\boldsymbol{H}^{(T) \stackrel{*}{=}} \frac{1}{4 \pi r c^{2}}\left[\hat{\boldsymbol{r}} \times \mathcal{F}+3\left(\frac{a}{c}\right) \hat{\boldsymbol{r}} \times \hat{\boldsymbol{r}} \times \hat{\boldsymbol{a}} \times \mathcal{Q}-\right. \\
\left.-3\left(\frac{a}{c}\right)^{2}(\hat{\boldsymbol{a}} \cdot \mathcal{G}) \hat{\boldsymbol{r}} \times \hat{\boldsymbol{a}}\right]_{\boldsymbol{T}=\boldsymbol{T}(t-r / c)}
\end{array}
$$

The electric $\left(\boldsymbol{E}^{(T)}\right)$ and magnetic fields $\left(\boldsymbol{H}^{(T)}\right)$ of the point-like particle, with toroidal dipole moment $\boldsymbol{T}$, that is momentarily at rest (at the origin) in the lab frame,
As in the case of electric dipole, the fields of the toroidal dipole are transverse, and are related through $\boldsymbol{H}^{(T)}=\frac{1}{\mu_{0} c} \hat{\boldsymbol{r}} \times \boldsymbol{E}^{(T)}$ indicating radial Poynting vector. 
C. Difference between a moving particle with (lab-frame) toroidization and particle with toroidal dipole

In the main text it is stated that properties of noninertial particles with net toroidal dipole moment have so far not been analyzed. Here we clarify this assertion. Work by J. A. Heras [36], has claimed to examine properties of non-inertial particles with toroidal dipole moment, but in fact considered properties of non-inertial particles which had net toroidization in the lab-frame. In this section we will briefly explain the important difference between a particle with toroidal dipole in its rest-frame, and a particle that appears to have net toroidal dipole in the lab-frame, i.e. some inertial frame around which we chose to base our calculations.

Clearly if a particle with toroidal dipole moment, described by current density in Eq. (5), is instantaneously at rest in the lab-frame, its current density is going to be that of a particle with toroidal dipole. It is tempting to assume that radiation produced by a particle with toroidal dipole, when it is instantaneously at rest in the lab frame, can be obtained by analyzing a particle with only the toroidization in the lab-frame, i.e. by using equations $\rho=0$ (charge density) and $\boldsymbol{J}=\nabla \times \nabla \times c \boldsymbol{T} \delta^{(3)}$, as it was done in Ref. [36]. This, however, is incorrect. Indeed, this is stated in Ref. [36].

As is shown in Eq. (B1), finding the far-field produced by a particle with toroidal dipole moment requires taking three derivatives with respect to proper time. Therefore, the instantaneous value of current density is insufficient to obtain radiation, instead one has to work with the full expression for the current density (Eq. (5)), and take the limit of particle being at rest in the lab frame after the electromagnetic fields have been found. Failing to do so results in some terms of the radiation field being lost without any justification. The origin of error is failure to take the derivatives of the basis vectors $\hat{\tau}^{\mu}=u^{\mu} / c\left(u^{\mu}\right.$ is the four-velocity of the particle) and $\hat{a}^{\mu}=a^{\mu} / \sqrt{-a \cdot a}$ ( $a^{\mu}$ is the four-acceleration of the particle).

\section{Inertial motion of an anapole}

The main focus of this paper is on anapole particles in non-inertial motion. It is natural to ask what happens in case of inertial motion. Here we briefly show that anapole in inertial motion does not radiate.

Given an anapole in inertial motion, one can start with its radiation in anapole particle's rest frame $\tilde{S}$. It follows from Eq. $(22,23)$, after substitution $a \rightarrow 0$ for inertial motion, that a non-accelerating anapole will emit no radiation in its rest frame (indeed this is the basic property of anapoles [8]). Therefore the corresponding electromagnetic tensor $\widetilde{F}_{\mu \eta}=0$. However since Maxwell Equations are invariant under (static) Lorentz transformation one can find the radiation in any other inertial frame, including lab-frame $S$, following:

$$
F_{\mu \eta}=\frac{\partial \tilde{x}^{\alpha}}{\partial x_{\mu}} \cdot \frac{\partial \tilde{x}^{\beta}}{\partial x_{\eta}} \cdot \tilde{F}_{\alpha \beta}=0
$$

The electromagnetic tensor $F_{\mu \eta}$ contains all components of electric and magnetic field, so if $F_{\mu \eta}=0$ then there is no radiation in the lab-frame. Therefore, anapoles in inertial motion do not radiate. 